\title{
On complexity of the quantum Ising model
}

\author{
Sergey Bravyi* $\quad$ Matthew Hastings ${ }^{\dagger}$
}

\begin{abstract}
We study complexity of several problems related to the Transverse field Ising Model (TIM). First, we consider the problem of estimating the ground state energy known as the Local Hamiltonian Problem (LHP). It is shown that the LHP for TIM on degree-3 graphs is equivalent modulo polynomial reductions to the LHP for general $k$-local 'stoquastic' Hamiltonians with any constant $k \geq 2$. This result implies that estimating the ground state energy of TIM on degree-3 graphs is a complete problem for the complexity class StoqMA - an extension of the classical class MA. As a corollary, we complete the complexity classification of 2-local Hamiltonians with a fixed set of interactions proposed recently by Cubitt and Montanaro. Secondly, we study quantum annealing algorithms for finding ground states of classical spin Hamiltonians associated with hard optimization problems. We prove that the quantum annealing with TIM Hamiltonians is equivalent modulo polynomial reductions to the quantum annealing with a certain subclass of $k$-local stoquastic Hamiltonians. This subclass includes all Hamiltonians representable as a sum of a $k$-local diagonal Hamiltonian and a 2-local stoquastic Hamiltonian.
\end{abstract}

${ }^{*}$ IBM T.J. Watson Research Center, Yorktown Heights, NY 10598, USA

${ }^{\dagger}$ Quantum Architectures and Computation Group, Microsoft Research, Redmond, WA 98052, USA 


\section{Contents}

1 Introduction and summary of results 3

2 Hard-core bosons and dimers $r$

3 Simulation of eigenvalues and eigenvectors 12

4 Schrieffer-Wolff transformation and perturbative reductions 15

5 Reduction from degree-3 graphs to general graphs 20

6 Reduction from TIM to dimers 23

7 Reduction from dimers to range-2 bosons 26

8 Range-2 bosons with multi-particle interactions 30

9 Reduction from range-2 bosons to range-1 bosons 32

10 Range-1 bosons with a controlled hopping 34

11 From range-1 bosons to 2-local stoquastic Hamiltonians 35

12 Proof of the main theorems $\quad 42$

A Bounds on the energy splitting and matrix elements for the Ising chain 44 


\section{Introduction and summary of results}

Numerical simulation of quantum many-body systems is a notoriously hard problem. A particularly strong form of hardness known as QMA-completeness [1] has been recently established for many natural problems in this category. Among them is the problem of estimating the ground state energy for certain physically-motivated quantum models such as Hamiltonians with nearest-neighbor interactions on the two-dimensional [2] and one-dimensional [3, 4] lattices, the Hubbard model [5, 6], and the Heisenberg model [5, 7]. In contrast, a broad class of Hamiltonians known as sign-free or stoquastic [8] has been identified for which certain simulation tasks become more tractable. By definition, stoquastic Hamiltonians must have real matrix elements with respect to some fixed basis and all off-diagonal matrix elements must be non-positive. Ground states of stoquastic Hamiltonians are known to have real non-negative amplitudes in the chosen basis. Thus, for many purposes, the ground state can be viewed as a classical probability distribution which often enables efficient simulation by quantum Monte Carlo algorithms $[9,10,11,12]$. A notable example of a model in this category is the transverse field Ising model (TIM). It has a Hamiltonian

$$
H=\sum_{1 \leq u \leq n} h_{u} X_{u}+g_{u} Z_{u}+\sum_{1 \leq u<v \leq n} g_{u, v} Z_{u} Z_{v}
$$

Here $n$ denotes the number of qubits (spins), $h_{u}, g_{u}, g_{u, v}$ are real coefficients, and $X_{u}, Z_{u}$ are the Pauli operators acting on a qubit $u$. Note that $H$ is a stoquastic Hamiltonian in the standard $Z$-basis iff $h_{u} \leq 0$ for all $u$. This can always be achieved by conjugating $H$ with $Z_{u}$. It is known that the ground state energy and the free energy of the TIM can be approximated with an additive error $\epsilon$ in time poly $\left(n, \epsilon^{-1}\right)$ using Monte Carlo algorithms [13] in the special case when the Ising interactions are ferromagnetic, that is, $g_{u, v} \leq 0$ for all $u, v$. Another important special case is the TIM defined on the one-dimensional lattice with $g_{u}=0$. In this case the Hamiltonian Eq. (1) is exactly solvable by the Jordan-Wigner transformation and its eigenvalues can be computed analytically [14]. The ground state and the thermal equilibrium properties of the TIM have been studied in many different contexts including quantum phase transitions [15], quantum spin glasses [16, 17] and quantum annealing algorithms [18, 19, 20, 21]. In the present paper we address two open questions related to the TIM. First, we consider the problem of estimating the ground state energy of the TIM and fully characterize its hardness in terms of the known complexity classes. Secondly we study quantum annealing algorithms with TIM Hamiltonians and show that such algorithms can efficiently simulate a much broader class of quantum annealing algorithms associated with many important classical optimization problems.

To state our main results let us define two classes of stoquastic Hamiltonians. Let $\operatorname{TIM}(n, J)$ be the set of all $n$-qubit transverse field Ising Hamiltonians defined in Eq. (1) such that the coefficients $h_{u}, g_{u}, g_{u, v}$ have magnitude at most $J$ for all $u, v$. A TIM Hamiltonian is said to have interactions of degree $d$ iff each qubit is coupled to at most $d$ other qubits with $Z Z$ interactions. Such Hamiltonian can be embedded into a degree- $d$ graph such that only nearest-neighbor qubits interact. We note that the terms of $H$ that are linear in $Z_{u}$ can be absorbed into the Ising interaction part by introducing 
one ancillary qubit $a$ and replacing $g_{u} Z_{u}$ by $g_{u} Z_{u} Z_{a}$ for each $u$. This transformation does not change the spectrum of $H$ except for doubling the multiplicity of each eigenvalue, see Section 2 for details.

Let StoqLH $(n, J)$ be the set of stoquastic 2-local Hamiltonians $H$ on $n$ qubits with the maximum interaction strength $J$. By definition, $H \in \operatorname{StoqLH}(n, J)$ iff

$$
H=\sum_{1 \leq u<v \leq n} H_{u, v}
$$

where $H_{u, v}$ is a hermitian operator acting on the qubits $u, v$ such that $\left\|H_{u, v}\right\| \leq J$ and all off-diagonal matrix elements of $H_{u, v}$ in the standard basis are real and non-positive. One can choose different operators $H_{u, v}$ for each pair of qubits. We shall provide a more explicit characterization of 2-local stoquastic Hamiltonians in terms of their Pauli expansion in Section 11, see Lemma 9.

Our first theorem asserts that any 2-local stoquastic Hamiltonian can appear as an effective low-energy theory emerging from the TIM on a degree-3 graph.

Theorem 1. Consider any Hamiltonian $H \in \operatorname{StoqLH}(n, J)$ and a precision parameter $\epsilon>0$. There exist $n^{\prime} \leq \operatorname{poly}(n), J^{\prime} \leq \operatorname{poly}\left(n, J, \epsilon^{-1}\right)$, and a Hamiltonian $H^{\prime} \in \operatorname{TIM}\left(n^{\prime}, J^{\prime}\right)$ such that

(1) The $i$-th smallest eigenvalues of $H$ and $H^{\prime}$ differ at most by $\epsilon$ for all $1 \leq i \leq 2^{n}$.

(2) One can compute $H^{\prime}$ in time poly $(n)$.

(3) $H^{\prime}$ has interactions of degree 3.

Here the maximum degree of all polynomial functions is some fixed constant that does not depend on any parameters (although we expect this constant to be quite large). The theorem has important implications for classifying complexity of the Local Hamiltonian Problem (LHP) [1, 22]. Recall that the LHP is a decision problem where one has to decide whether the ground state energy $E_{0}$ of a given Hamiltonian $H$ acting on $n$ qubits is sufficiently small, $E_{0} \leq E_{\text {yes }}$, or sufficiently large, $E_{0} \geq E_{n o}$. Here $E_{y e s}<E_{n o}$ are some specified thresholds such that $E_{n o}-E_{y e s} \geq \operatorname{poly}\left(n^{-1}\right)$. The Hamiltonian must be representable as a sum of hermitian operators acting on at most $k$ qubits each, where $k=O(1)$ is a small constant. Each $k$-qubit operator must have norm at most poly $(n)$. Such Hamiltonians are known as $k$-local. Theorem 1 implies that the LHP for 2-local stoquastic Hamiltonians has the same complexity as the LHP for TIM. Indeed, consider an instance of the LHP for some Hamiltonian $H \in \operatorname{StoqLH}(n, J)$ where $J \leq \operatorname{poly}(n)$. Choose a precision $\epsilon=\left(E_{n o}-E_{\text {yes }}\right) / 3$ and let $H^{\prime}$ be the TIM Hamiltonian constructed in Theorem 1. Note that $H^{\prime}$ acts on poly $(n)$ qubits and has the interaction strength poly $(n)$. Let $E_{0}^{\prime}$ be the ground state energy of $H^{\prime}$. Then $E_{0} \leq E_{\text {yes }}$ implies $E_{0}^{\prime} \leq E_{\text {yes }}+\epsilon \equiv E_{\text {yes }}^{\prime}$ and $E_{0} \geq E_{n o}$ implies $E_{0}^{\prime} \geq E_{n o}-\epsilon \equiv E_{n o}^{\prime}$. Since $E_{n o}^{\prime}-E_{\text {yes }}^{\prime}=\left(E_{n o}-E_{\text {yes }}\right) / 3 \geq \operatorname{poly}\left(n^{-1}\right)$, the LHP for a 2-local stoquastic Hamiltonian has been reduced to the LHP for TIM. The converse reduction is trivial since any TIM Hamiltonian can be made stoquastic by a local change of basis. Thus we obtain

Corollary 1. The LHP for 2-local stoquastic Hamiltonians has the same complexity as the LHP for TIM with interactions of degree 3 , modulo polynomial reductions. 
It is known that the LHPs for 2-local and $k$-local stoquastic Hamiltonians have the same complexity for any constant $k \geq 2$, modulo polynomial reductions [8]. Thus estimating the ground state energy of TIM on a degree-3 graph is as hard as estimating the ground state energy of a general $k$-local stoquastic Hamiltonian for $k=O(1)$. Furthermore, the LHP for 6-local stoquastic Hamiltonians is known to be a complete problem for the complexity class StoqMA [8, 23]. This is an extension of the classical class MA where the verifier can accept quantum states as a proof. To examine the proof the verifier is allowed to apply classical reversible gates in a coherent fashion and, finally, measure some fixed qubit in the $X$-basis. The verifier accepts the proof if the measurement outcome is ' + '. Let $P_{a c c}(x)$ be the acceptance probability of the verifier for a given problem instance $x$ maximized over all possible proofs. A decision problem belongs to StoqMA if there exist a polynomial-size verifier as above and threshold probabilities $P_{y e s} \geq P_{n o}+\operatorname{poly}\left(n^{-1}\right)$ such that $P_{a c c}(x) \geq P_{y e s}$ for any yes-instance $x$ and $P_{a c c}(x) \leq P_{n o}$ for any no-instance $x$. Here $n$ is the length of the problem instance $x$, see [23] for a formal definition. Combining these known results and Corollary 1 we obtain

Corollary 2. The Local Hamiltonian Problem for TIM with interactions of degree 3 is complete for the complexity class StoqMA.

Finally, Theorem 1 completes the complexity classification of 2-local Hamiltonians with a fixed set of interactions proposed recently by Cubitt and Montanaro [7]. The problem studied in [7] is defined as follows. Let $\mathcal{S}$ be a fixed set of two-qubit hermitian operators. Consider a special case of the 2-local LHP such that Hamiltonians are required to have a form $H=\sum_{a} x_{a} V_{a}$, where $x_{a}$ is a real coefficient and $V_{a}$ is an operator from $\mathcal{S}$ applied to some pair of qubits. For brevity, let us call the above problem $\mathcal{S}$-LHP. The main result of Ref. [7] is that depending on the choice of $\mathcal{S}$, the problem $\mathcal{S}$-LHP is either complete for one of the complexity classes NP, QMA, or can be solved in polynomial time on a classical computer, or can be reduced in polynomial time to the LHP for TIM. In addition, one can efficiently determine which case is realized for a given choice of $\mathcal{S}$. Combining this result and Corollary 2 one obtains

Corollary 3. Let $\mathcal{S}$ be any fixed set of two-qubit hermitian operators. Then depending on $\mathcal{S}$, the problem $\mathcal{S}$-LHP is either complete for one of the complexity classes NP, StoqMA, QMA, or can be solved in polynomial time on a classical computer.

We also prove an analogue of Theorem 1 which gives new insights on the power of quantum annealing (QA) algorithms [18, 24] with TIM Hamiltonians which received a significant attention recently [19, 20, 21]. Recall that quantum annealing (QA) [18, 24] attempts to find a global minimum of a real-valued function $f\left(x_{1}, \ldots, x_{n}\right)$ that depends on $n$ binary variables by encoding $f$ into a diagonal problem Hamiltonian $H_{P}=\sum_{x} f(x)|x\rangle\langle x|$ acting on $n$ qubits. To find the ground state of $H_{P}$ one chooses an adiabatic path $H(\tau)=(1-\tau) H(0)+\tau H_{P}, 0 \leq \tau \leq 1$, where $H(0)$ is some simple Hamiltonian usually chosen as the transverse magnetic field, $H(0)=-\sum_{u=1}^{n} X_{u}$. Initializing the system in the ground state of $H(0)$ and traversing the adiabatic path slowly enough one can approximately prepare the ground state of $H_{P}$. The running time of QA algorithms scales as poly $\left(n, \delta^{-1}\right)$, where $\delta$ is the minimum spectral gap of $H(\tau)$, see $[18,24,25]$. We focus on the special case of QA 
such that the objective function $f\left(x_{1}, \ldots, x_{n}\right)$ is a sum of terms that depend on at most $k$ variables each. Here $k=O(1)$ is some small constant. This includes well-known optimization problems such as $k$-SAT, MAX- $k$-SAT and many variations thereof. We show that any quantum annealing algorithm as above can be efficiently simulated by the quantum annealing with TIM Hamiltonians. The simulation has a slowdown at most poly $\left(n, \delta^{-1}\right)$.

Fix some integer $k \geq 2$. We will say that $H$ is a $(2, k)$-local stoquastic Hamiltonian iff $H$ is a sum of a 2-local stoquastic Hamiltonian and a $k$-local diagonal Hamiltonian. Let StoqLH ${ }^{*}(n, J)$ be the set of all $(2, k)$-local stoquastic Hamiltonians on $n$-qubits with the maximum interaction strength $J$.

Theorem 2. Consider any Hamiltonian $H \in \operatorname{StoqLH}^{*}(n, J)$ with a non-degenerate ground state $|g\rangle$ and a spectral gap $\delta$. There exist $n^{\prime} \leq \operatorname{poly}(n), J^{\prime} \leq \operatorname{poly}\left(n, J, \delta^{-1}\right)$, a Hamiltonian $H^{\prime} \in \operatorname{TIM}\left(n^{\prime}, J^{\prime}\right)$, and an isometry $\mathcal{E}:\left(\mathbb{C}^{2}\right)^{\otimes n} \rightarrow\left(\mathbb{C}^{2}\right)^{\otimes n^{\prime}}$ such that

(1) $H^{\prime}$ has a non-degenerate ground state $\left|g^{\prime}\right\rangle$ and a spectral gap at least $\delta / 3$.

(2) $\|\left|g^{\prime}\right\rangle-\mathcal{E}|g\rangle \| \leq 1 / 100$.

(3) The isometry $\mathcal{E}$ maps basis vectors to basis vectors.

(4) One can compute $H^{\prime}$ and the action of $\mathcal{E}, \mathcal{E}^{\dagger}$ on any basis vector in time poly $(n)$.

Here the maximum degree of all polynomial functions depends only on the locality parameter $k$. We note that one can replace the constant 1/100 in condition (2) by an arbitrary precision parameter $\eta>0$. Then the same theorem holds with a scaling $J^{\prime} \leq \operatorname{poly}\left(n, J, \delta^{-1}, \eta^{-1}\right)$. One can also impose a restriction that the Hamiltonian $H^{\prime}$ has interactions of degree-3. Then a similar theorem holds, but the isometry $\mathcal{E}$ has slightly more complicated properties, see Section 12 for details.

Let us discuss implications of the theorem. Suppose $H(\tau) \in \operatorname{StoqLH}^{*}(n, J)$ is an adiabatic path such that $H(1)=H_{P}$ is the problem Hamiltonian and $H(0)=-\sum_{u=1}^{n} X_{u}$. We assume that $H(\tau)$ has a non-degenerate ground state $|g(\tau)\rangle$ and a spectral gap at least $\delta$ for all $\tau$. Also we assume that $J \leq$ poly $(n)$. Since $X_{u}$ can be adiabatically rotated to $Z_{u}$ without closing the gap, we can modify the path such that $H(0)=-\sum_{u=1}^{n} Z_{u}$. Then the initial ground state is $|g(0)\rangle=\left|0^{\otimes n}\right\rangle$. Applying Theorem 2 to each Hamiltonian $H(\tau)$ one obtains a family of TIM Hamiltonians $H^{\prime}(\tau)$ such that $H^{\prime}(\tau)$ has a non-degenerate ground state $\left|g^{\prime}(\tau)\right\rangle \approx \mathcal{E}|g(\tau)\rangle$, the spectral gap at least $\delta / 3$, and the interaction strength at most poly $\left(n, \delta^{-1}\right)$. We will show that the map $H \rightarrow H^{\prime}$ is sufficiently smooth, so that the family $H^{\prime}(\tau), 0 \leq \tau \leq 1$, defines an adiabatic path and the time it takes to traverse the paths $H(\tau)$ and $H^{\prime}(\tau)$ differ at most by a factor poly $\left(n, \delta^{-1}\right)$, see Section 12 for details. Therefore one can (approximately) prepare the final state $\left|g^{\prime}(1)\right\rangle$ by initializing the system in the basis state $\mathcal{E}\left|0^{\otimes n}\right\rangle \approx\left|g^{\prime}(0)\right\rangle$ and traversing the path $H^{\prime}(\tau)$. Measuring every qubit of the final state $\left|g^{\prime}(1)\right\rangle$ in the $Z$-basis one obtains a string of outcomes $x \in\{0,1\}^{n^{\prime}}$ such that $\mathcal{E}|g(1)\rangle \approx|x\rangle$. Then $|g(1)\rangle \approx \mathcal{E}^{\dagger}|x\rangle$, that is, the ground state of $H_{P}$ can be efficiently computed from $x$. Thus we obtain

Corollary 4. Any quantum annealing algorithm with $(2, k)$-local stoquastic Hamiltonians can be simulated by a quantum annealing algorithm with TIM Hamiltonians. The simulation has overhead at most poly $\left(n, \delta^{-1}\right)$, where $n$ is the number of qubits and $\delta$ is the minimum spectral gap of the adiabatic path. 
In the rest of this section we informally sketch the proof of the main theorems, discuss several open problems, and outline organization of the paper.

Sketch of the proof. The proof of Theorems 1,2 relies on perturbative reductions [22, 2] and the Schrieffer-Wolff transformation [26, 27, 28]. At each step of the proof we work with two quantum models: a simulator Hamiltonian $H_{\text {sim }}$ acting on some Hilbert space $\mathcal{H}$ and a target Hamiltonian $H_{\text {target }}$ acting on a certain subspace ${ }^{1} \mathcal{H}_{-} \subseteq \mathcal{H}$. We represent $\mathcal{H}_{-}$as the low-energy subspace of a suitable Hamiltonian $H_{0}$ which has a large energy gap $\Delta$ for all eigenvectors orthogonal to $\mathcal{H}_{-}$. We choose $H_{\text {sim }}=H_{0}+V$, where $V$ is a weak perturbation such that $\|V\| \ll \Delta$. We show that $H_{\text {target }}$ can be obtained from $H_{\text {sim }}$ as an effective low-energy Hamiltonian calculated using a few lowest orders of the perturbation theory. More precisely, $H_{\text {target }} \approx P_{-} U\left(H_{0}+V\right) U^{\dagger} P_{-}$, where $P_{-}$is the projector onto $\mathcal{H}_{-}$and $U$ is a unitary operator on $\mathcal{H}$ known as the Schrieffer-Wolff transformation. The latter brings $H_{0}+V$ into a block-diagonal form such that $U\left(H_{0}+V\right) U^{\dagger}$ preserves the subspace $\mathcal{H}_{-}$. We show that the low-lying eigenvalues and eigenvectors of $H_{\text {sim }}$ approximate the respective eigenvalues and eigenvectors of $H_{\text {target }}$ with an error that can made arbitrarily small by choosing large enough $\Delta$. We apply the above step recursively several times such that the target Hamiltonian at the $t$-th step

\begin{tabular}{|c|}
\hline TIM, degree-3 graph \\
\hline TIM, general graph \\
\hline Hard-core dimers, triangle-free graph \\
\hline Hard-core bosons, range-2 \\
\hline Hard-core bosons, range-1 \\
\hline Hard-core bosons, range-1, controlled hopping \\
\hline 2-local stoquastic Hamiltonians \\
\hline
\end{tabular}

Table 1: Perturbative reductions used in the proof of Theorem 1. Each model is obtained as an effective low-energy Hamiltonian for the model located one row above. The hard-core bosons (HCB) model describes a multi-particle quantum walk on a graph. The Hamiltonian consists of a hopping term, on-site chemical potential, and arbitrary two-particle interactions. Different particles must be separated from each other by a certain minimum distance that we call a range of the model. HCB is closely related to the Bose-Hubbard model. The hard-core dimers model is analogous to HCB except that admissible particle configuration must consist of nearest-neighbor pairs of particles that we call dimers. Different dimers must be separated from each other by a certain minimum distance. A rigorous definition of the models is given in Section 2.

becomes the simulator Hamiltonian at the $(t+1)$-th step. The recursion starts from the TIM with interactions of degree- 3 at the highest energy scale, goes through several intermediate models listed in Table 1 , and arrives at a given 2-local or $(2, k)$-stoquastic Hamiltonian at the lowest energy scale. Overall, the proof requires nine different reductions ${ }^{2}$. To simplify the analysis of recursive reductions

\footnotetext{
${ }^{1}$ More precisely, we identify $H_{\text {target }}$ with a Hamiltonian acting on the subspace $\mathcal{H}_{-}$using a suitable encoding.

${ }^{2}$ Some of our reductions are 'trivial' in the sense that they simply restrict a Hamiltonian to a certain subspace.
} 
we introduce a general definition of a simulation that quantifies how close are two different models in terms of their low-lying eigenvalues and eigenvectors. Our definition is shown to be stable under the composition of simulations.

For almost all of our reductions the Hamiltonian $H_{0}$ is diagonal in the standard basis, so that all eigenvalues and eigenvectors of $H_{0}$ can be easily computed. The only exception is the reduction from TIM with interactions of degree-3 to a general TIM. For this reduction we encode each qubit of the target model into the approximately two-fold degenerate ground subspace of the one-dimensional TIM on a chain of a suitable length. Accordingly, the Hamiltonian $H_{0}$ describes a collection of onedimensional TIMs. We simulate the logical Ising interaction $Z_{u} Z_{v}$ between some pair of logical qubits $u, v$ by applying the physical interaction $Z_{i} Z_{j}$ to a properly chosen pair of qubits $i \in L_{u}$ and $j \in L_{v}$, where $L_{u}$ is the chain encoding a logical qubit $u$. The logical transverse field $X_{u}$ is automatically generated due to the energy splitting between the ground states of $L_{u}$. The analysis of this reduction exploits recent exact results on the form-factors of the one-dimensional TIM [29].

We emphasize that the word "reduction" is used in two distinct senses. In the present paper we speak of a perturbative reduction from a Hamiltonian $H_{\text {sim }}$ to a Hamiltonian $H_{\text {target }}$ when $H_{\text {target }}$ is the effective low-energy Hamiltonian derived from $H_{\text {sim }}$, following terminology in physics. However, if $H_{\text {target }}$ belongs to some particular class of Hamiltonians $\mathcal{T}$ and $H_{\text {sim }}$ belongs to some subclass $\mathcal{S} \subseteq \mathcal{T}$, this is a reduction from the class $\mathcal{T}$ to the class $\mathcal{S}$, according to terminology in computer science.

Open problems. Our work raises several questions. First, we expect that Theorems 1,2 can be extended in a number of ways. For example, one may ask whether the analogue of Theorem 1 holds for TIM Hamiltonians restricted to particular families of graphs, such as planar graphs or regular lattices. We note that a simple modification of our degree reduction method based on the one-dimensional TIM produces a simulator Hamiltonian which can be embedded into the 3D lattice of dimensions $n \times n \times 2$ with periodic boundary conditions. We expect that applying additional perturbative reductions such as those described in Ref. [2] can further simplify the lattice. Likewise, we expect that Theorem 2 can be extended to the case when $H$ is a general $k$-local stoquastic Hamiltonian by applying perturbative reductions of Ref. [8].

A challenging open question is whether TIM Hamiltonians defined on a 2D lattice can realize the topological quantum order. It has been recently shown that the hard-core bosons model defined on the kagome lattice has a topologically ordered ground state for a certain range of parameters [30, 31]. A preliminary analysis shows that the chain of reductions from TIM to hard-core bosons described in the present paper can be modified such that all intermediate Hamiltonians have geometrically local interactions. Assuming that the unphysical polynomial scaling of interactions in the simulator Hamiltonian can be avoided [27, 32], this points towards existence of topologically ordered phases described by TIM Hamiltonians.

Finally, a big open question is whether QA algorithms with TIM Hamiltonians can be efficiently simulated classically. It has been recently shown that the general purpose quantum Monte Carlo algorithms fail to simulate certain instances of the QA with TIM efficiently [33], even though these instances have a non-negligible minimum spectral gap. This leaves a possibility that some more

The proof contains only six 'non-trivial' reductions that actually change the Hamiltonian. 
specialized algorithms taking advantage of the special structure of TIM Hamiltonians can succeed even though the general purpose algorithm fail. Our results demonstrate that this is unlikely, since simulating the QA with TIM is as hard as simulating the QA with much more general $(2, k)$-local stoquastic Hamiltonians.

The paper is organized as follows. Section 2 contains a rigorous definition of the models listed in Table 1. Our main technical tools are introduced in Sections 3,4 which present a general definition of a simulation, describe perturbative reductions based on the Schrieffer-Wolff transformation, and prove several technical lemmas used in the rest of the paper. Section 5 shows how to simulate a general TIM Hamiltonian using a special case of TIM with interactions of degree-3. Sections 6-11 describe a chain of perturbative reductions between the models listed in Table 1. These reductions are combined together in Section 12 which contains the proof of Theorems 1,2. Finally, Appendix A proves certain bounds on eigenvalues and form-factors of the one-dimensional TIM which are used in Section 5.

\section{Hard-core bosons and dimers}

Consider a graph $G=(U, E)$ with a set of $n$ nodes $U$ and a set of edges $E$. Define a Hilbert space $\mathcal{B} \cong\left(\mathbb{C}^{2}\right)^{\otimes n}$ with an orthonormal basis $\{|S\rangle: S \subseteq U\}$ such that basis vectors are labeled by subsets

of nodes $S$. We shall identify subsets of nodes with configurations of particles that live at nodes of the graph. Each node can be either empty or occupied by a single particle. For any node $u \in U$ define a particle number operator $n_{u}$ such that $n_{u}|S\rangle=|S\rangle$ if $u \in S$ and $n_{u}|S\rangle=0$ otherwise. We shall often consider diagonal Hamiltonians of the following form:

$$
H_{\text {diag }}=\sum_{u \in U} \mu_{u} n_{u}+\sum_{\{u, v\} \subseteq U} \omega_{u, v} n_{u} n_{v} .
$$

Here the second sum runs over all two-node subsets (not only nearest neighbors). The coefficients $\mu_{u}$ and $\omega_{u, v}$ can be viewed as a chemical potential and a two-particle interaction potential respectively.

Let us now define a hopping operator $W_{u, v}$. Here $u, v \in U$ are arbitrary nodes such that $u \neq v$. By definition, $W_{u, v}$ annihilates any state $|S\rangle$ in which both nodes $u, v$ are occupied or both nodes are empty. If one of the nodes $u, v$ is occupied and the other node is empty, $W_{u, v}$ transfers a particle from $u$ to $v$ or vice verse. Matrix elements of $W_{u, v}$ in the chosen basis are

$$
\left\langle S^{\prime}\left|W_{u, v}\right| S\right\rangle=\left\{\begin{array}{lll}
1 & \text { if } & u \in S, v \notin S, \text { and } S^{\prime}=(S \backslash u) \cup v \\
1 & \text { if } \quad v \in S, u \notin S, \text { and } S^{\prime}=(S \backslash v) \cup u \\
0 & & \text { otherwise. }
\end{array}\right.
$$

Let $m, r \geq 1$ be fixed integers. Define a subspace $\mathcal{B}_{m} \subset \mathcal{B}$ spanned by all subsets $S \subseteq U$ with exactly $m$ nodes. We shall refer to $\mathcal{B}_{m}$ as an $m$-particle sector. Obviously, the operators $W_{u, v}$ and $n_{u}$ preserve $\mathcal{B}_{m}$. A subset of nodes $S$ is said to be $r$-sparse iff the graph distance between any distinct pair of nodes $u, v \in S$ is at least $r$. Define a subspace $\mathcal{B}_{m, r} \subseteq \mathcal{B}_{m}$ spanned by all $r$-sparse subsets 
$S \subseteq U$ with exactly $m$ nodes. By definition, any subset of nodes is 1 -sparse, so that $\mathcal{B}_{m, 1}=\mathcal{B}_{m}$. Note that the operators $W_{u, v}$ generally do not preserve $\mathcal{B}_{m, r}$. Below we consider hopping operators $W_{u, v}$ projected onto the subspace $\mathcal{B}_{m, r}$. Matrix elements of a projected hopping operator are defined by Eq. (3), where $S$ and $S^{\prime}$ run over all $r$-sparse subsets of $m$ nodes.

Our first model is called hard-core bosons (HCB). It is defined on the Hilbert space $\mathcal{B}_{m, r}$, where $m$ and $r$ are fixed parameters. We shall refer to $r$ as the range of the model. The Hamiltonian is

$$
H=-\sum_{(u, v) \in E} t_{u, v} W_{u, v}+H_{\text {diag }} .
$$

Here $H_{\text {diag }}$ is defined by Eq. (2) and all operators are projected onto the subspace $\mathcal{B}_{m, r}$. Thus $W_{u, v}$ moves a particle only if this does not violate the $r$-sparsity condition. Otherwise $W_{u, v}$ annihilates a state. The coefficients $t_{u, v}$ are hopping amplitudes. We shall always assume that

$$
t_{u, v} \geq 0
$$

for all $u, v$. The coefficients $\mu_{u}$ and $\omega_{u, v}$ in $H_{\text {diag }}$ may have arbitrary signs. Note that $H$ is a stoquastic Hamiltonian. Let $\mathrm{HCB}_{r}(n, m, J)$ be the set of Hamiltonians describing the $m$-particle sector of range$r$ hard-core bosons on a graph with $n$ nodes such that all the coefficients $\mu_{u}, \omega_{u, v}, t_{u, v}$ have magnitude at most $J$. Here we take the union over all graphs $G$ with $n$ nodes. Our proof will only use HCB models with the range $r=1,2$. Later on we shall define certain enhanced versions of the HCB which have multi-particle interactions, see Section 8, and/or controlled hopping terms, see Section 10. We note that the HCB model with non-positive hopping amplitudes $t_{u, v} \leq 0$ has been recently studied by Childs, Gosset, and Webb [6] who showed that the corresponding LHP is QMA-complete.

Our second model is called hard-core dimers. This model also depends on a graph $G=(U, E)$. We shall only consider triangle-free graphs $G$. Let $m \geq 1$ be a fixed integer parameter. A subset of nodes $S \subseteq U$ is said to be a dimer iff $S=\{u, v\}$ for some pair of nodes $u \neq v$ such that $(u, v) \in E$. Define an $m$-dimer as a subset of nodes $S \subseteq U$ that can be represented as a disjoint union of $m$ dimers $S_{1}, \ldots, S_{m}$ such that the graph distance between $S_{i}$ and $S_{j}$ is at least three for all $i \neq j$. This particular choice of the distance guarantees that $m$-dimers can be represented as ground states of a suitable Ising Hamiltonian, see Lemma 8 in Section 6. Examples of 2-dimers are shown on Fig. 1.
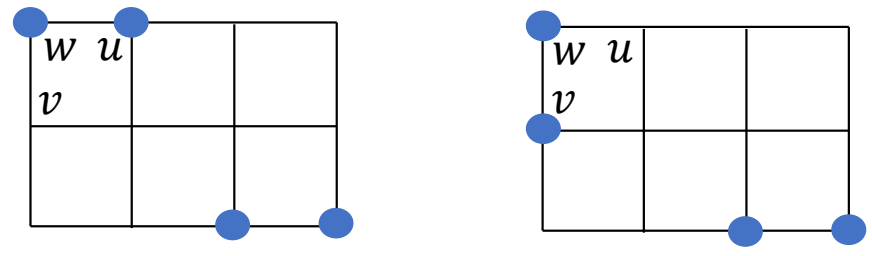

Figure 1: Examples of 2-dimers $S, S^{\prime}$ on the square grid such that $\left|S^{\prime}\right\rangle=W_{u, v}|S\rangle$.

Let $\mathcal{D}_{m} \subseteq \mathcal{B}_{2 m}$ be the subspace spanned by all basis vectors $|S\rangle$ such that $S \subseteq U$ is an $m$-dimer. Note that the operators $W_{u, v}$ generally do not preserve $\mathcal{D}_{m}$. Below we consider hopping operators 
$W_{u, v}$ projected onto the subspace $\mathcal{D}_{m}$. Matrix elements of a projected hopping operator are defined by Eq. (3), where $S$ and $S^{\prime}$ run over all $m$-dimers. The hard-core dimers (HCD) model has a Hilbert space $\mathcal{D}_{m}$ and a Hamiltonian

$$
H=-t \sum_{\{u, v\} \subseteq U} W_{u, v}+H_{\text {diag }}
$$

where $H_{\text {diag }}$ is defined by Eq. (2) and all operators are projected onto the subspace $\mathcal{D}_{m}$. The sum in Eq. (5) runs over all pairs of nodes (not only nearest neighbors). Although the Hamiltonian does not explicitly depend on the graph structure, the underlying Hilbert space $\mathcal{D}_{m}$ does depends on the graph since the latter determines which subsets of nodes are $m$-dimers. A hopping process induced by $W_{u, v}$ can change a dimer $\{w, u\}$ to some other dimer $\{w, v\}$ with $u \neq v$, see Fig. 1 . The coefficient $t$ is a hopping amplitude. We shall assume that $t \geq 0$. Then $H$ is a stoquastic Hamiltonian. Let $\operatorname{HCD}(n, m, J)$ be the set of Hamiltonians describing the $m$-dimer sector of hard-core dimers model on a graph with $n$ nodes such that all coefficients in $H$ have magnitude at most $J$. Here we take the union over all triangle-free graphs with $n$ nodes.

Some perturbative reductions described below will alter the underlying graph $G$. Whenever the choice of $G$ is not clear from the context, we shall use more detailed notations $\mathcal{B}_{m}(G), \mathcal{B}_{m, r}(G)$, and $\mathcal{D}_{m}(G)$ instead of $\mathcal{B}_{m}, \mathcal{B}_{m, r}$, and $\mathcal{D}_{m}$. Our notations for various classes of Hamiltonians are summarized in Table 2.

\begin{tabular}{c|c}
\hline $\operatorname{TIM}(n, J)$ & Transverse field Ising Model \\
\hline $\operatorname{HCD}(n, m, J)$ & $m$-dimer sector of Hard-Core Dimers model. \\
\hline $\operatorname{HCB}_{r}(n, m, J)$ & $m$-particle sector of Hard-Core Bosons with range $r$. \\
\hline $\operatorname{HCB}^{*}(n, m, J)$ & same as $\mathrm{HCB}_{1}(n, m, J)$. \\
\hline $\operatorname{HCB}^{*}(n, m, J)$ & $\operatorname{HCB}(n, m, J)$ with controlled hopping terms. \\
\hline StoqLH $(n, J)$ & Stoquastic 2-Local Hamiltonians. \\
\hline
\end{tabular}

Table 2: Classes of Hamiltonians used in the proof of Theorem 1. Here $J$ denotes the maximum interaction strength and $n$ denotes the number of nodes in the graph (the number of qubits). For those models that depend on a graph, the corresponding class is defined by taking the union over all graphs with a fixed number of nodes $n$ (all triangle-free graphs in the case of HCD). The class HCB* is formally defined in Section 10.

Finally, consider a TIM Hamiltonian $H \in \operatorname{TIM}(n, J)$ defined in Eq. (1). Let us add an ancillary qubit labeled by ' $a$ ' and consider a modified Hamiltonian $H^{\prime} \in \operatorname{TIM}(n+1, J)$ defined as

$$
H^{\prime}=\sum_{u=1}^{n} h_{u} X_{u}+g_{u} Z_{u} Z_{a}+\sum_{1 \leq u<v \leq n} g_{u, v} Z_{u} Z_{v} .
$$

Let $X_{\text {all }}=X^{\otimes(n+1)}$ be the global spin flip operator. Note that $H$ commutes with $Z_{a}$ and $X_{\text {all }}$, whereas $Z_{a}$ and $X_{\text {all }}$ anti-commute. This implies that the restriction of $H^{\prime}$ onto the sectors $Z_{a}=1$ 
and $Z_{a}=-1$ have exactly the same spectrum as the original Hamiltonian $H$. Hence the full spectrum of $H^{\prime}$ is obtained from the one of $H$ by doubling the multiplicity of each eigenvalue. In particular, the LHPs for Hamiltonians Eqs. $(1,6)$ have the same complexity. Finally, substituting $Z_{u}=I-2 n_{u}$ into Eq. (1) one gets

$$
H=\sum_{u \in U} \mu_{u} n_{u}+\sum_{\{u, v\} \subseteq U} \omega_{u, v} n_{u} n_{v}+h_{u} X_{u}=H_{\text {diag }}+\sum_{u \in U} h_{u} X_{u}
$$

where $U \equiv\{1, \ldots, n\}, \omega_{u, v}=4 g_{u, v}$ and $\mu_{u}=-2 g_{u}-2 \sum_{v \neq u} g_{u, v}$. Here we ignore the overall energy shift. Clearly, the coefficients $\omega_{u, v}$ and $\mu_{u}$ have magnitude at most $O(n J)$. Below we shall work with TIM Hamiltonians as defined in Eq. (7).

\section{Simulation of eigenvalues and eigenvectors}

In this section we give a formal definition of a simulation. It quantifies how close are two different models in terms of their low-energy properties such as the low-lying eigenvalues and eigenvectors. We consider a target model described by a Hamiltonian $H$ acting on some $N$-dimensional Hilbert space $\mathcal{H}$ and a simulator model described by a Hamiltonian $H_{\text {sim }}$ acting on some Hilbert space $\mathcal{H}_{\text {sim }}$ of dimension at least $N$. Our definition of a simulation depends on a particular encoding transformation $\mathcal{E}: \mathcal{H} \rightarrow \mathcal{H}_{\text {sim }}$ that embeds $\mathcal{H}$ into some $N$-dimensional subspace of $\mathcal{H}_{\text {sim }}$. We assume that $\mathcal{E}$ is an isometry, that is, $\mathcal{E}^{\dagger} \mathcal{E}=I$. The encoding enables a comparison between eigenvectors of the two models. We envision a situation when the spectrum of $H_{\text {sim }}$ consists of two well-separated groups of eigenvalues such that the $N$ smallest eigenvalues of $H_{\text {sim }}$ are separated from the rest of its spectrum by a large gap. Let $\mathcal{L}_{N}\left(H_{\text {sim }}\right) \subseteq \mathcal{H}_{\text {sim }}$ be the low-energy subspace spanned by the eigenvectors of $H_{\text {sim }}$ associated with its $N$ smallest eigenvalues.

Definition 1. Let $H$ be a Hamiltonian acting on a Hilbert space $\mathcal{H}$ of dimension $N$. A Hamiltonian $H_{\text {sim }}$ and an isometry (encoding) $\mathcal{E}: \mathcal{H} \rightarrow \mathcal{H}_{\text {sim }}$ are said to simulate $H$ with an error $(\eta, \epsilon)$ if there exists an isometry $\tilde{\mathcal{E}}: \mathcal{H} \rightarrow \mathcal{H}_{\text {sim }}$ such that

S1. The image of $\tilde{\mathcal{E}}$ coincides with the low-energy subspace $\mathcal{L}_{N}\left(H_{\text {sim }}\right)$.

S2. $\left\|H-\tilde{\mathcal{E}}^{\dagger} H_{\operatorname{sim}} \tilde{\mathcal{E}}\right\| \leq \epsilon$.

S3. $\|\mathcal{E}-\tilde{\mathcal{E}}\| \leq \eta$.

Although we do not impose any restrictions on the encoding, in practice it must be sufficiently simple. For all our reductions (except for the one of Section 5) the encoding $\mathcal{E}$ maps basis vectors to basis vectors. Whenever the choice of $\mathcal{E}$ is clear from the context, we shall just say that $H_{\text {sim }}$ simulates $H$ with an error $(\eta, \epsilon)$. If one is interested only in reproducing eigenvalues of the target Hamiltonian, the encoding and condition (S3) can be ignored.

In the case of a zero error, $\epsilon=\eta=0$, the target Hamiltonian $H$ coincides with the restriction of $H_{\text {sim }}$ onto the low-energy subspace of $H_{\text {sim }}$, up to a change of basis described by $\mathcal{E}$. Clearly, any 
Hamiltonian simulates itself with a zero error since one can choose $\mathcal{E}=\tilde{\mathcal{E}}=I$. We shall always assume that $\epsilon \leq\|H\|$ since otherwise the definition is meaningless (one can choose $H_{\text {sim }}=0$ regardless of $H)$. Note that $\epsilon$ has the dimension of energy while $\eta$ is dimensionless. Loosely speaking, $\epsilon$ and $\eta$ quantify simulation error for eigenvalues and eigenvectors respectively. Let us establish some basic properties of simulations.

Lemma 1 (Eigenvalue simulation). Suppose $\left(H_{\text {sim }}, \mathcal{E}\right)$ simulates $H$ with an error $(\eta, \epsilon)$. Then the $i$-th smallest eigenvalues of $H_{\operatorname{sim}}$ and $H$ differ at most by $\epsilon$ for all $1 \leq i \leq N$.

Proof. Property (S1) implies that the spectrum of $\tilde{\mathcal{E}}^{\dagger} H_{\text {sim }} \tilde{\mathcal{E}}$ coincides with $N$ smallest eigenvalues of $H_{\text {sim. }}$. The lemma now follows from (S2) and the standard Weyl's inequality.

Lemma 2 (Ground state simulation). Suppose $H$ has a non-degenerate ground state $|g\rangle$ separated from excited states by a spectral gap $\delta$. Suppose $\left(H_{\text {sim }}, \mathcal{E}\right)$ simulates $H$ with an error $(\eta, \epsilon)$ such that $2 \epsilon<\delta$. Then $H_{\text {sim }}$ has a non-degenerate ground state $\left|g_{\text {sim }}\right\rangle$ and

$$
\| \mathcal{E}|g\rangle-\left|g_{\text {sim }}\right\rangle \| \leq \eta+O\left(\delta^{-1} \epsilon\right)
$$

Proof. Let $\left|g_{\text {sim }}\right\rangle$ be the ground state of $H_{\text {sim }}$. Note that $\left|g_{\text {sim }}\right\rangle$ is non-degenerate due to Lemma 1 and the assumption $2 \epsilon<\delta$. Consider an unperturbed Hamiltonian $H$ and a perturbation $V=$ $\tilde{\mathcal{E}}^{\dagger} H_{\text {sim }} \tilde{\mathcal{E}}-H$. The perturbed Hamiltonian $H+V=\tilde{\mathcal{E}}^{\dagger} H_{\text {sim }} \tilde{\mathcal{E}}$ has a non-degenerate ground state $\tilde{\mathcal{E}}^{\dagger}\left|g_{\text {sim }}\right\rangle$. Using the first-order perturbation theory for eigenvectors one gets $\||g\rangle-\tilde{\mathcal{E}}^{\dagger}\left|g_{\text {sim }}\right\rangle \| \leq O\left(\delta^{-1} \epsilon\right)$ and thus $\| \tilde{\mathcal{E}}|g\rangle-\left|g_{\text {sim }}\right\rangle\|=\| \tilde{\mathcal{E}}|g\rangle-\tilde{\mathcal{E}} \tilde{\mathcal{E}}^{\dagger}\left|g_{\text {sim }}\right\rangle \| \leq O\left(\delta^{-1} \epsilon\right)$. Here we used the fact that $\tilde{\mathcal{E}}$ is an isometry and $\left|g_{\text {sim }}\right\rangle \in \mathcal{L}_{N}\left(H_{\text {sim }}\right)=\operatorname{Im}(\tilde{\mathcal{E}})$. Property (S3) then leads to Eq. (8).

Importantly, our definition of a simulation is stable under compositions: if one is given some Hamiltonians $H, H_{1}, H_{2}$ such that $H_{1}$ simulates $H$ with a small error and $H_{2}$ simulates $H_{1}$ with a small error, this implies that $H_{2}$ simulates $H$ with a small error.

Lemma 3 (Composition). Suppose $\left(H_{1}, \mathcal{E}_{1}\right)$ simulates $H$ with an error $\left(\eta_{1}, \epsilon_{1}\right)$ and $\left(H_{2}, \mathcal{E}_{2}\right)$ simulates $H_{1}$ with an error $\left(\eta_{2}, \epsilon_{2}\right)$. Let $\Delta_{1}$ be the spectral gap separating $N$ smallest eigenvalues of $H_{1}$ from the rest of the spectrum. Suppose $2 \epsilon_{2}<\Delta_{1}$ and $\epsilon_{1}, \epsilon_{2} \leq\|H\|$. Then $\left(H_{2}, \mathcal{E}_{2} \mathcal{E}_{1}\right)$ simulates $H$ with an error $(\eta, \epsilon)$, where

$$
\eta=\eta_{1}+\eta_{2}+O\left(\epsilon_{2} \Delta_{1}^{-1}\right) \quad \text { and } \quad \epsilon=\epsilon_{1}+\epsilon_{2}+O\left(\epsilon_{2} \Delta_{1}^{-1}\|H\|\right) .
$$

We shall always choose the simulator such that $\Delta_{1} \gg\|H\|$ in which case $\epsilon \approx \epsilon_{1}+\epsilon_{2}$.

Proof. Suppose $H, H_{1}, H_{2}$ act on Hilbert spaces $\mathcal{H}, \mathcal{H}_{1}, \mathcal{H}_{2}$ respectively. Let $N=\operatorname{dim}(\mathcal{H})$ and $N_{1}=\operatorname{dim}\left(\mathcal{H}_{1}\right)$. By Lemma 1 , the $N$ smallest eigenvalues of $H_{2}$ are separated from the rest of the spectrum by a spectral gap at least $\Delta_{1}-2 \epsilon_{2}>0$. Thus the low-energy subspace $\mathcal{L}_{N}\left(H_{2}\right)$ is well defined. Let $\tilde{\mathcal{E}}_{2}: \mathcal{H}_{1} \rightarrow \mathcal{H}_{2}$ be an isometry satisfying properties (S1-S3) for a simulator $\left(H_{2}, \mathcal{E}_{2}\right)$ and a target Hamiltonian $H_{1}$ with an error $\left(\eta_{2}, \epsilon_{2}\right)$. By definition, $\tilde{\mathcal{E}}_{2}$ maps $\mathcal{H}_{1}$ to the low-energy subspace 
$\mathcal{L}_{N_{1}}\left(H_{2}\right)$. First, let us show that $\tilde{\mathcal{E}}_{2}$ approximately maps $\mathcal{L}_{N}\left(H_{1}\right)$ to $\mathcal{L}_{N}\left(H_{2}\right)$. More precisely, we claim that there exists a unitary operator $U: \mathcal{L}_{N_{1}}\left(\mathcal{H}_{2}\right) \rightarrow \mathcal{L}_{N_{1}}\left(\mathcal{H}_{2}\right)$ such that

$$
\mathcal{L}_{N}\left(H_{2}\right)=U \tilde{\mathcal{E}}_{2} \cdot \mathcal{L}_{N}\left(H_{1}\right) \text { and } \quad\|U-I\| \leq 2 \sqrt{2} \Delta_{1}^{-1} \epsilon_{2}
$$

Indeed, let $P_{N}\left(H_{i}\right)$ be the projector onto the low-energy subspace $\mathcal{L}_{N}\left(H_{i}\right)$, where $i=1$, 2. Consider a pertubation $V=\tilde{\mathcal{E}}_{2}^{\dagger} H_{2} \tilde{\mathcal{E}}_{2}-H_{1}$. Note that $\mathcal{L}_{N}\left(H_{1}+V\right)=\tilde{\mathcal{E}}_{2}^{\dagger} \cdot \mathcal{L}_{N}\left(H_{2}\right)$. Applying Lemma 3.1 of Ref. [28] with an unperturbed Hamiltonian $H_{1}$ and a perturbation $V$ one gets

$$
\left\|P_{N}\left(H_{1}\right)-\tilde{\mathcal{E}}_{2}^{\dagger} P_{N}\left(H_{2}\right) \tilde{\mathcal{E}}_{2}\right\| \leq \frac{2\left\|\tilde{\mathcal{E}}_{2}^{\dagger} H_{2} \tilde{\mathcal{E}}_{2}-H_{1}\right\|}{\Delta_{1}} \leq \frac{2 \epsilon_{2}}{\Delta_{1}} .
$$

Taking into account that $\mathcal{L}_{N}\left(H_{2}\right) \subseteq \mathcal{L}_{N_{1}}\left(H_{2}\right)=\operatorname{Im}\left(\tilde{\mathcal{E}}_{2}\right)$ one gets

$$
\left\|\tilde{\mathcal{E}}_{2} P_{N}\left(H_{1}\right) \tilde{\mathcal{E}}_{2}^{\dagger}-P_{N}\left(H_{2}\right)\right\|=\left\|P_{N}\left(H_{1}\right)-\tilde{\mathcal{E}}_{2}^{\dagger} P_{N}\left(H_{2}\right) \tilde{\mathcal{E}}_{2}\right\|
$$

and thus

$$
\left\|\tilde{\mathcal{E}}_{2} P_{N}\left(H_{1}\right) \tilde{\mathcal{E}}_{2}^{\dagger}-P_{N}\left(H_{2}\right)\right\| \leq \frac{2 \epsilon_{2}}{\Delta_{1}}
$$

For brevity denote

$$
P \equiv \tilde{\mathcal{E}}_{2} P_{N}\left(H_{1}\right) \tilde{\mathcal{E}}_{2}^{\dagger} \quad \text { and } \quad Q \equiv P_{N}\left(H_{2}\right) \text {. }
$$

By Jordan's lemma, there exists an orthonormal basis such that the projectors $P$ and $Q$ are blockdiagonal in this basis with all blocks being either $1 \times 1$ or $2 \times 2$ projectors. Assuming that $2 \Delta_{1}^{-1} \epsilon_{2}<1$ one has $\|P-Q\|<1$ which implies that all $1 \times 1$ blocks of $P$ and $Q$ are the same. Consider some $2 \times 2$ block. Without loss of generality, the restrictions of $P$ and $Q$ onto this block have a form

$$
P=\left[\begin{array}{ll}
1 & 0 \\
0 & 0
\end{array}\right] \text { and } Q=\left[\begin{array}{cc}
c^{2} & c s \\
c s & s^{2}
\end{array}\right]
$$

for some $0 \leq c, s \leq 1$ such that $c^{2}+s^{2}=1$. Then $P-Q=s^{2} Z-c s X$ and thus $\|P-Q\|=s$. We conclude that $s \leq 2 \Delta_{1}^{-1} \epsilon_{2}$ for any $2 \times 2$ block. Define a unitary

$$
U=\left[\begin{array}{cc}
c & -s \\
s & c
\end{array}\right]=c I-i s Y
$$

such that $U P U^{\dagger}=Q$. Note that $\|U-I\|=|c-1+i s| \leq \sqrt{2} s$. Extending $U$ to the full space $\mathcal{L}_{N_{1}}\left(\mathcal{H}_{2}\right)$ we obtain $\operatorname{Im}(Q)=U \cdot \operatorname{Im}(P)$ and $\|U-I\| \leq \sqrt{2} s$ which is equivalent to Eq. (10).

Now we are ready to prove that $\left(H_{2}, \mathcal{E}_{2} \mathcal{E}_{1}\right)$ simulates $H$ with a small error. Define an isometry

$$
\tilde{\mathcal{E}}=U \tilde{\mathcal{E}}_{2} \tilde{\mathcal{E}}_{1}
$$

Using the first part of Eq. (10) and the fact that $\tilde{\mathcal{E}}_{1}$ maps $\mathcal{H}$ to the low-energy subspace $\mathcal{L}_{N}\left(H_{1}\right)$ we conclude that $\tilde{\mathcal{E}}$ maps $\mathcal{H}$ to the low-energy subspace $\mathcal{L}_{N}\left(H_{2}\right)$. Thus $\tilde{\mathcal{E}}$ obeys property (S1) for 
the target Hamiltonian $H$ and the simulator $\left(H_{2}, \mathcal{E}_{2} \mathcal{E}_{1}\right)$. Furthermore, the second part of Eq. (10) implies that

$$
\eta \equiv\left\|\tilde{\mathcal{E}}-\mathcal{E}_{2} \mathcal{E}_{1}\right\| \leq\|U-I\|+\left\|\tilde{\mathcal{E}}_{2}-\mathcal{E}_{2}\right\|+\left\|\tilde{\mathcal{E}}_{1}-\mathcal{E}_{1}\right\| \leq 2 \sqrt{2} \Delta_{1}^{-1} \epsilon_{2}+\eta_{2}+\eta_{1}
$$

Finally, let $H_{i}^{(N)}=H_{i} P_{N}\left(H_{i}\right)$ be the restriction of $H_{i}$ onto the low-energy subspace $\mathcal{L}_{N}\left(H_{i}\right)$. Note that $H_{1} \tilde{\mathcal{E}}_{1}=H_{1}^{(N)} \tilde{\mathcal{E}}_{1}$ and $H_{2} U \tilde{\mathcal{E}}_{2} \tilde{\mathcal{E}}_{1}=H_{2}^{(N)} U \tilde{\mathcal{E}}_{2} \tilde{\mathcal{E}}_{1}$. Thus

$$
\left\|H-\tilde{\mathcal{E}}^{\dagger} H_{2} \tilde{\mathcal{E}}\right\| \leq\left\|H-\tilde{\mathcal{E}}_{1}^{\dagger} H_{1} \tilde{\mathcal{E}}_{1}\right\|+\left\|\tilde{\mathcal{E}}_{1}^{\dagger}\left(H_{1}^{(N)}-\tilde{\mathcal{E}}_{2}^{\dagger} U^{\dagger} H_{2}^{(N)} U \tilde{\mathcal{E}}_{2}\right) \tilde{\mathcal{E}}_{1}\right\|
$$

The first term is upper bounded by $\epsilon_{1}$. Thus

$$
\left\|H-\tilde{\mathcal{E}}^{\dagger} H_{2} \tilde{\mathcal{E}}\right\| \leq \epsilon_{1}+\left\|U \tilde{\mathcal{E}}_{2} H_{1}^{(N)}-H_{2}^{(N)} U \tilde{\mathcal{E}}_{2}\right\| .
$$

To bound the second term we write $U=I+M$ and note that

$$
\begin{aligned}
U \tilde{\mathcal{E}}_{2} H_{1}^{(N)}-H_{2}^{(N)} U \tilde{\mathcal{E}}_{2} & =P_{N}\left(H_{2}\right)\left(U \tilde{\mathcal{E}}_{2} H_{1}-H_{2} U \tilde{\mathcal{E}}_{2}\right) P_{N}\left(H_{1}\right) \\
& =P_{N}\left(H_{2}\right)\left(\tilde{\mathcal{E}}_{2} H_{1}-H_{2} \tilde{\mathcal{E}}_{2}\right) P_{N}\left(H_{1}\right)+P_{N}\left(H_{2}\right) M \tilde{\mathcal{E}}_{2} H_{1}^{(N)}-H_{2}^{(N)} M \tilde{\mathcal{E}}_{2} P_{N}\left(H_{1}\right) .
\end{aligned}
$$

The norm of the first term is upper bounded by

$$
\left\|\tilde{\mathcal{E}}_{2} H_{1}-H_{2} \tilde{\mathcal{E}}_{2}\right\| \leq\left\|H_{1}-\tilde{\mathcal{E}}_{2}^{\dagger} H_{2} \tilde{\mathcal{E}}_{2}\right\| \leq \epsilon_{2}
$$

Thus

$$
\left\|H-\tilde{\mathcal{E}}^{\dagger} H_{2} \tilde{\mathcal{E}}\right\| \leq \epsilon_{1}+\epsilon_{2}+\|U-I\| \cdot\left\|H_{1}^{(N)}\right\|+\|U-I\| \cdot\left\|H_{2}^{(N)}\right\| .
$$

Lemma 1 implies that $\left\|H_{2}^{(N)}\right\| \leq\left\|H_{1}^{(N)}\right\|+\epsilon_{2}$ and $\left\|H_{1}^{(N)}\right\| \leq\|H\|+\epsilon_{1}$. Combining this and the second part of Eq. (10) one arrives at

$$
\left\|H-\tilde{\mathcal{E}}^{\dagger} H_{2} \tilde{\mathcal{E}}\right\| \leq \epsilon_{1}+\epsilon_{2}+O\left(\Delta_{1}^{-1} \epsilon_{2}\|H\|\right)+O\left(\Delta_{1}^{-1}\left(\epsilon_{2}^{2}+\epsilon_{1} \epsilon_{2}\right)\right) .
$$

Since we assumed that $\epsilon_{1}, \epsilon_{2} \leq\|H\|$, the last term is at most $O\left(\Delta_{1}^{-1} \epsilon_{2}\|H\|\right)$.

\section{Schrieffer-Wolff transformation and perturbative reduc- tions}

Let $H$ be a target Hamiltonian chosen from some particular class of Hamiltonians $\mathcal{C}$. Suppose our goal is to simulate $H$ with a small error according to Definition 1 using a simulator Hamiltonian $H_{\text {sim }}$ which is required to be a member of some smaller class $\mathcal{C}^{\prime} \subset \mathcal{C}$. Perturbative reductions [22, 2] provide a general method of accomplishing such simulation. Here we describe perturbative reductions based on the Schrieffer-Wolff transformation [26], see for instance [28] and the references therein. Also we provide sufficient conditions under which a $k$-th order reduction achieves the desired simulation error for $k=1,2,3$, see Lemmas 4-7. 
Consider a finite-dimensional Hilbert space $\mathcal{H}_{\text {sim }}$ decomposed into a direct sum

$$
\mathcal{H}_{\text {sim }}=\mathcal{H}_{-} \oplus \mathcal{H}_{+}
$$

Let $N_{ \pm}=\operatorname{dim}\left(\mathcal{H}_{ \pm}\right)$and $P_{ \pm}$be the projector onto $\mathcal{H}_{ \pm}$such that $P_{-}+P_{+}=I$. Let $O$ be any linear operator on $\mathcal{H}_{\text {sim }}$. We shall write

$$
O_{--}=P_{-} O P_{-}, \quad O_{-+}=P_{-} O P_{+}, \quad O_{+-}=P_{+} O P_{-}, \quad O_{++}=P_{+} O P_{+} .
$$

The operator is said to be block diagonal if $O_{-+}=0$ and $O_{+-}=0$. The operator is said to be block off-diagonal if $O_{--}=0$ and $O_{++}=0$.

Let $H_{0}$ and $V$ be hermitian operators on $\mathcal{H}_{\text {sim }}$ such that $H_{0}$ is block-diagonal, $\left(H_{0}\right)_{--}=0$, and such that $\left(H_{0}\right)_{++}$has all eigenvalues greater or equal to one. Consider a perturbed Hamiltonian

$$
H_{\text {sim }}=\Delta H_{0}+V
$$

where $\Delta$ is a large parameter. We shall always assume that

$$
\|V\|<\Delta / 2
$$

The Schrieffer-Wolff transformation is a unitary operator on $\mathcal{H}_{\text {sim }}$ defined as $e^{S}$, where $S$ is an antihermitian operator satisfying

$$
\left(e^{S} H_{\text {sim }} e^{-S}\right)_{-+}=0, \quad\left(e^{S} H_{\text {sim }} e^{-S}\right)_{+-}=0, \quad S_{--}=0, \quad S_{++}=0, \quad\|S\|<\pi / 2 .
$$

In other words, we require that the transformed Hamiltonian $e^{S} H_{\text {sim }} e^{-S}$ is block diagonal whereas $S$ itself is block off-diagonal. It is known that Eq. (16) has a unique solution $S$, see Lemma 2.3 and Lemma 3.1 in [28]. In particular, $S=0$ if $V=0$. The effective low-energy Hamiltonian $H_{\text {eff }}$ is a hermitian operator acting on $\mathcal{H}_{-}$defined as

$$
H_{\text {eff }}=\left(e^{S} H_{\text {sim }} e^{-S}\right)_{--} .
$$

Note that $H_{\text {eff }}=0$ if $V=0$. Since the operator $e^{S}$ is unitary and the transformed Hamiltonian $e^{S} H_{\text {sim }} e^{-s}$ is block-diagonal, each eigenvalue of $H_{\text {eff }}$ must be an eigenvalue of $H_{\text {sim }}$. It is known that the $i$-th smallest eigenvalues of $H_{\text {eff }}$ and $H_{\text {sim }}$ coincide for all $1 \leq i \leq N_{-}$, see [28] for details.

Consider now the Taylor series $S=\sum_{j=1}^{\infty} S_{j}$ and $H_{\mathrm{eff}}=\sum_{j=1}^{\infty} H_{\mathrm{eff}, j}$, where $S_{j}$ and $H_{\mathrm{eff}, j}$ are the Taylor coefficients proportional to the $j$-th power of $V$. We shall only need the Taylor coefficients

$$
\begin{gathered}
H_{\text {eff }, 1}=V_{--}, \\
H_{\text {eff }, 2}=-\Delta^{-1} V_{-+} H_{0}^{-1} V_{+-}
\end{gathered}
$$

and

$$
H_{\mathrm{eff}, 3}=\Delta^{-2} V_{-+} H_{0}^{-1} V_{++} H_{0}^{-1} V_{+-}-\frac{\Delta^{-2}}{2}\left(V_{-+} H_{0}^{-2} V_{+-} V_{--}+\text {h.c. }\right),
$$


see Section 3.2 of [28] for the derivation. Note that the restriction of $H_{0}^{-1}$ to the subspace $\mathcal{H}_{+}$is well-defined since all eigenvalues of $\left(H_{0}\right)_{++}$are at least one. It is known that the series for $S$ and $H_{\text {eff }}$ converge absolutely for $\|V\|<\Delta / 16$ and the $j$-th Taylor coefficients are bounded as

$$
\left\|S_{j}\right\| \leq(b \Delta)^{-j}\|V\|^{j} \quad \text { and } \quad\left\|H_{\mathrm{eff}, j}\right\| \leq(c \Delta)^{1-j}\|V\|^{j}
$$

for some constant coefficients $b, c>0$, see Lemma 3.4 in [28]. Define the $k$-th order effective Hamiltonian as the truncated series

$$
H_{\mathrm{eff}}(k)=\sum_{j=1}^{k} H_{\mathrm{eff}, j} .
$$

The above implies that for any $k=O(1)$ and $\|V\|<\Delta \cdot \min \{b, c\}$ one has

$$
\|S\| \leq \sum_{j=1}^{\infty}(b \Delta)^{-j}\|V\|^{j}=O\left(\Delta^{-1}\|V\|\right)
$$

and

$$
\left\|H_{\mathrm{eff}}-H_{\mathrm{eff}}(k)\right\| \leq \sum_{j=k+1}^{\infty}(c \Delta)^{1-j}\|V\|^{j}=O\left(\Delta^{-k}\|V\|^{k+1}\right) .
$$

Suppose now that $H_{\text {target }}$ is a fixed target Hamiltonian acting on some Hibert space $\mathcal{H}_{\text {target }}$ and $\mathcal{E}: \mathcal{H}_{\text {target }} \rightarrow \mathcal{H}_{\text {sim }}$ is some fixed isometry (encoding) such that $\operatorname{Im}(\mathcal{E})=\mathcal{H}_{-}$. Define a logical target Hamiltonian acting on $\mathcal{H}_{-}$as

$$
\bar{H}_{\text {target }}=\mathcal{E} H_{\text {target }} \mathcal{E}^{\dagger}
$$

The goal of perturbative reductions is to approximate $\bar{H}_{\text {target }}$ by the effective low-energy Hamiltonian $H_{\text {eff }}(k)$ emerging from the simulator Hamiltonian $H_{\text {sim }}$ defined in Eq. (14), where the parameter $\Delta$ controls the approximation error. Below we outline a general strategy for constructing the simulator Hamiltonian proposed by Oliveira and Terhal [2]. The strategy depends on the order $k$ of a reduction.

For first-order reductions one just needs to choose $V$ such that $\bar{H}_{\text {target }}=(V)_{--}$, see Eq. (17). For second-order reductions the perturbation $V$ will be chosen as

$$
V=\Delta^{1 / 2} V_{\text {main }}+V_{\text {extra }}
$$

where $\left(V_{\text {main }}\right)_{--}=0$ and $V_{\text {extra }}$ is block-diagonal. Both operators $V_{\text {main }}$ and $V_{\text {extra }}$ are independent of $\Delta$. Substituting $V$ into Eqs. $(17,18)$ gives

$$
H_{\text {eff }, 1}=\left(V_{\text {extra }}\right)_{--} \quad \text { and } \quad H_{\text {eff }, 2}=-\left(V_{\text {main }}\right)_{-+} H_{0}^{-1}\left(V_{\text {main }}\right)_{+-} .
$$

We shall choose $V_{\text {main }}$ such that $H_{\text {eff,2 }}$ generates the desired logical target Hamiltonian and, may be, some unwanted terms. The purpose of $V_{\text {extra }}$ is to cancel the unwanted terms. In addition, $V_{\text {extra }}$ may include all the terms of the target Hamiltonian that are members of the simulator class, such as two-qubit diagonal interactions. Note that the latter belong to all the classes listed in Table 2. Most of the second-order reductions described below will achieve an exact equality $H_{\text {eff }}(2)=\bar{H}_{\text {target }}$. 
For third-order reductions the perturbation $V$ will be chosen as

$$
V=\Delta^{2 / 3} V_{\text {main }}+\Delta^{1 / 3} \tilde{V}_{\text {extra }}+V_{\text {extra }}
$$

where $\left(V_{\text {main }}\right)_{--}=0$, and $V_{\text {extra }}, \tilde{V}_{\text {extra }}$ are block-diagonal. All operators $V_{\text {main }}, V_{\text {extra }}$, and $\tilde{V}_{\text {extra }}$ are independent of $\Delta$. Substituting $V$ into Eqs. (17-19) gives

$$
H_{\text {eff }, 1}=\Delta^{1 / 3}\left(\tilde{V}_{\text {extra }}\right)_{--}+\left(V_{\text {extra }}\right)_{--}, \quad H_{\text {eff }, 2}=-\Delta^{1 / 3}\left(V_{\text {main }}\right)_{-+} H_{0}^{-1}\left(V_{\text {main }}\right)_{+-},
$$

and

$$
H_{\text {eff }, 3}=\left(V_{\text {main }}\right)_{-+} H_{0}^{-1}\left(V_{\text {main }}\right)_{++} H_{0}^{-1}\left(V_{\text {main }}\right)_{+-}+O\left(\Delta^{-1 / 3}\left\|\tilde{V}_{\text {extra }}\right\| \cdot\left\|V_{\text {main }}\right\|^{2}\right) .
$$

We shall choose $\Delta$ large enough so that the last term in Eq. (29) can be neglected. We shall chose $V_{\text {main }}$ such that $H_{\text {eff,3 }}$ generates the desired logical target Hamiltonian and, may be, some unwanted terms. The purpose of $V_{\text {extra }}$ is to cancel the unwanted terms. In addition, $V_{\text {extra }}$ may include all the terms of the target Hamiltonian that are members of the simulator class. Finally, the purpose of

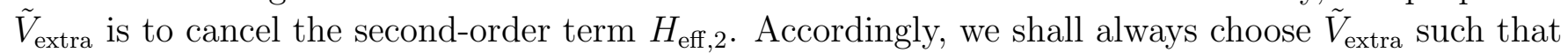

$$
\left(\tilde{V}_{\text {extra }}\right)_{--}=\left(V_{\text {main }}\right)_{-+} H_{0}^{-1}\left(V_{\text {main }}\right)_{+-} \text {. }
$$

Our proof will only use reductions of the order $k=1,2,3$. The following lemmas provide sufficient conditions under which a $k$-th order reduction achieves a desired simulation error. Recall that our definition of a simulation depends on a particular encoding $\mathcal{E}$, see Definition 1 . The lemmas stated below apply to any fixed encoding $\mathcal{E}$ and the logical target Hamiltonian defined by Eq. (24). In Lemmas 4-6 we assume that $H_{0}$ is block-diagonal, $\left(H_{0}\right)_{--}=0$, and $\left(H_{0}\right)_{++}$has all eigenvalues greater or equal to 1 .

Lemma 4 (First-order reduction). Suppose one can choose $H_{0}, V$ such that

$$
\left\|\bar{H}_{\text {target }}-(V)_{--}\right\| \leq \epsilon / 2 .
$$

Then $H_{\text {sim }}=\Delta H_{0}+V$ simulates $H_{\text {target }}$ with an error $(\eta, \epsilon)$, provided that $\Delta \geq O\left(\epsilon^{-1}\|V\|^{2}+\eta^{-1}\|V\|\right)$.

Proof. Let $\mathcal{E}: \mathcal{H}_{\text {target }} \rightarrow \mathcal{H}_{\text {sim }}$ be the chosen encoding. Recall that $\operatorname{Im}(\mathcal{E})=\mathcal{H}_{-}$. Let us check that $\tilde{\mathcal{E}}=e^{-S} \mathcal{E}$ satisfies conditions (S1-S3) of Definition 1. By definition of the Schrieffer-Wolff transformation, $e^{-S}$ maps $\mathcal{H}_{-}$to the low-energy subspace of $H_{\text {sim }}$. Thus $\tilde{\mathcal{E}}$ maps $\mathcal{H}_{\text {target }}$ to the low-energy subspace of $H_{\text {sim }}$ which proves (S1). From Eq. (23) one infers that $\left\|H_{\text {eff }}-H_{\text {eff }}(1)\right\| \leq$ $O\left(\Delta^{-1}\|V\|^{2}\right) \leq \epsilon / 2$. Combining this and Eq. (31) gives $\left\|\bar{H}_{\text {target }}-H_{\text {eff }}\right\| \leq \epsilon$ and thus $\| H_{\text {target }}-$ $\mathcal{E}^{\dagger} H_{\text {eff }} \mathcal{E} \| \leq \epsilon$. Substituting $H_{\text {eff }}=\left(e^{S} H_{\text {sim }} e^{-S}\right)_{--}$one gets $\left\|H_{\text {target }}-\tilde{\mathcal{E}}^{\dagger} H_{\text {sim }} \tilde{\mathcal{E}}\right\| \leq \epsilon$ which proves condition (S2). Finally, Eq. (22) leads to $\|\mathcal{E}-\tilde{\mathcal{E}}\|=\left\|I-e^{-S}\right\|=O(\|S\|)=O\left(\Delta^{-1}\|V\|\right) \leq \eta$. This proves condition (S3).

Lemma 5 (Second-order reduction). Suppose one can choose $H_{0}, V_{\text {main }}, V_{\text {extra }}$ such that $V_{\text {extra }}$ is block-diagonal, $\left(V_{\text {main }}\right)_{--}=0$, and

$$
\left\|\bar{H}_{\text {target }}-\left(V_{\text {extra }}\right)_{--}+\left(V_{\text {main }}\right)_{-+} H_{0}^{-1}\left(V_{\text {main }}\right)_{+-}\right\| \leq \epsilon / 2 .
$$

Suppose the norm of $V_{\text {main }}, V_{\text {extra }}$ is at most $\Lambda$. Then $H_{\text {sim }}=\Delta H_{0}+\Delta^{1 / 2} V_{\text {main }}+V_{\text {extra }}$ simulates $H_{\text {target }}$ with an error $(\eta, \epsilon)$ provided that $\Delta \geq O\left(\epsilon^{-2} \Lambda^{6}+\eta^{-2} \Lambda^{2}\right)$. 
Proof. Let $V=\Delta^{1 / 2} V_{\text {main }}+V_{\text {extra. }}$. By assumption, $V$ has norm $O\left(\Delta^{1 / 2} \Lambda\right)$. Substituting this into Eq. (23) gives $\left\|H_{\text {eff }}-H_{\text {eff }}(2)\right\| \leq O\left(\Delta^{-2}\|V\|^{3}\right)=O\left(\Delta^{-1 / 2} \Lambda^{3}\right) \leq \epsilon / 2$. From Eqs. (26,32) one gets $\left\|\bar{H}_{\text {target }}-H_{\text {eff }}(2)\right\| \leq \epsilon / 2$ which gives $\left\|H_{\text {eff }}-\bar{H}_{\text {target }}\right\| \leq \epsilon$. Finally, Eq. (22) leads to $\|\mathcal{E}-\tilde{\mathcal{E}}\|=$ $\left\|I-e^{-S}\right\|=O(\|S\|)=O\left(\Delta^{-1}\|V\|\right)=O\left(\Delta^{-1 / 2} \Lambda\right) \leq \eta$. The rest of the proof is identical to the one of Lemma 4.

Lemma 6 (Third-order reduction). Suppose one can choose $H_{0}, V_{\text {main }}, V_{\text {extra }}, \tilde{V}_{\text {extra }}$ such that $V_{\text {extra }}, \tilde{V}_{\text {extra }}$ are block-diagonal, $\left(V_{\text {main }}\right)_{--}=0$,

$$
\left\|\bar{H}_{\text {target }}-\left(V_{\text {extra }}\right)_{--}-\left(V_{\text {main }}\right)_{-+} H_{0}^{-1}\left(V_{\text {main }}\right)_{++} H_{0}^{-1}\left(V_{\text {main }}\right)_{+-}\right\| \leq \epsilon / 2,
$$

and

$$
\left(\tilde{V}_{\text {extra }}\right)_{--}=\left(V_{\text {main }}\right)_{-+} H_{0}^{-1}\left(V_{\text {main }}\right)_{+-} .
$$

Suppose the norm of $V_{\text {main }}, V_{\text {extra }}, \tilde{V}_{\text {extra }}$ is at most $\Lambda$. Then $H_{\text {sim }}=\Delta H_{0}+\Delta^{2 / 3} V_{\text {main }}+\Delta^{1 / 3} \tilde{V}_{\text {extra }}+V_{\text {extra }}$ simulates $H_{\text {target }}$ with an error $(\eta, \epsilon)$ provided that $\Delta \geq O\left(\epsilon^{-3} \Lambda^{12}+\eta^{-3} \Lambda^{3}\right)$.

Proof. Let $V=\Delta^{2 / 3} V_{\text {main }}+\Delta^{1 / 3} \tilde{V}_{\text {extra }}+V_{\text {extra. }}$. By assumption, $V$ has norm $O\left(\Delta^{2 / 3} \Lambda\right)$. Substituting this into Eq. (23) gives $\left\|H_{\text {eff }}-H_{\text {eff }}(3)\right\| \leq O\left(\Delta^{-3}\|V\|^{4}\right)=O\left(\Delta^{-1 / 3} \Lambda^{4}\right) \leq \epsilon / 4$. Combining Eqs. $(28,29)$ and Eqs. $(33,34)$ one gets

$$
\left\|\bar{H}_{\text {target }}-H_{\text {eff }}(3)\right\| \leq \epsilon / 4+O\left(\Delta^{-1 / 3}\left\|\tilde{V}_{\text {extra }}\right\| \cdot\left\|V_{\text {main }}\right\|^{2}\right)=\epsilon / 4+O\left(\Delta^{-1 / 3} \Lambda^{3}\right) \leq \epsilon / 2 .
$$

This gives $\left\|H_{\text {eff }}-\bar{H}_{\text {target }}\right\| \leq \epsilon$. Finally, Eq. (22) leads to $\|\mathcal{E}-\tilde{\mathcal{E}}\|=\left\|I-e^{-S}\right\|=O(\|S\|)=$ $O\left(\Delta^{-1}\|V\|\right)=O\left(\Delta^{-1 / 3} \Lambda\right) \leq \eta$. The rest of the proof is identical to the one of Lemma 4.

The above framework covers all our reductions except for the one described in Section 5, namely, the reduction from TIM on degree-3 graphs to TIM on general graphs. The latter is a first-order reduction where the Hamiltonian $H_{0}$ is chosen as the the one-dimensional TIM. In this case $H_{0}$ has only approximately degenerate ground subspace, $\left(H_{0}\right)_{--} \neq 0$, and the rules Eq. (17-19) for computing the effective Hamiltonian no longer apply. The following is a simple generalization of Lemma 4.

Lemma 7 (Generalized first-order reduction). Suppose one can choose $H_{0}, V$ such that $H_{0}$ is block-diagonal, $\left(H_{0}\right)_{++}$has all eigenvalues at least $\Delta$, and $\left(H_{0}\right)_{--}$has all eigenvalues in the interval $[-\Delta / 2, \Delta / 2]$. Suppose also that

$$
\left\|\bar{H}_{\text {target }}-\left(H_{0}\right)_{--}-(V)_{--}\right\| \leq \epsilon / 2 .
$$

Then $H_{\mathrm{sim}}=H_{0}+V$ simulates $H_{\text {target }}$ with an error $(\eta, \epsilon)$ provided that $\Delta \geq O\left(\epsilon^{-1}\|V\|^{2}+\eta^{-1}\|V\|\right)$.

Proof. We can use the same arguments as in the proof of Lemma 4 except that now $H_{\text {eff }}(1)=H_{\text {eff, } 0}+$ $H_{\text {eff, }, 1}$, where $H_{\text {eff }, 0}=\left(H_{0}\right)_{--}$and $H_{\text {eff, }, 1}=(V)_{--}$. By assumption, the unperturbed Hamiltonian $H_{0}$ has an energy gap at least $\Delta / 2$ separating $\mathcal{H}_{-}$and $\mathcal{H}_{+}$. Lemma 3.4 of Ref. [28] implies that the

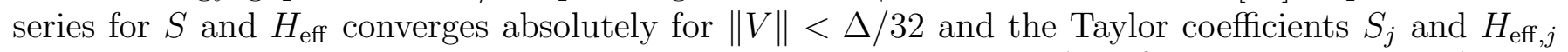
are bounded as in Eq. $(22,23)$. Therefore $\left\|H_{\text {eff }}-H_{\text {eff }}(1)\right\| \leq O\left(\Delta^{-1}\|V\|^{2}\right)$ and $\|S\| \leq O\left(\Delta^{-1}\|V\|\right)$. The rest of the proof is the same as in Lemma 4. 


\section{Reduction from degree-3 graphs to general graphs}

Consider a target Hamiltonian $H_{\text {target }}$ describing the TIM on $n$ qubits. We assume that each qubit can be coupled to any other qubit with $Z Z$ interactions. Below we show how to simulate $H_{\text {target }}$ using TIM with interactions of degree 3 . Let us first informally sketch the main idea. We shall encode each qubit $u$ of the target model into the ground subspace of a TIM Hamiltonian on a one-dimensional chain $L_{u}$ of some length $m$. The chain will be in the ferromagnetic phase such that the ground states $\psi_{0}$ and $\psi_{1}$ originating from the two different $\mathbb{Z}_{2}$-symmetry sectors are approximately degenerate forming one logical qubit. The basis states of the logical qubit will be defined as $|\overline{0}\rangle \sim\left|\psi_{0}\right\rangle+\left|\psi_{1}\right\rangle$ and $|\overline{1}\rangle \sim\left|\psi_{0}\right\rangle-\left|\psi_{1}\right\rangle$. Important parameters of the logical qubit are the energy splitting $\delta$ between $\psi_{0}$ and $\psi_{1}$ and the energy gap $\Delta$ separating $\psi_{0}, \psi_{1}$ from excited states. We shall work in the regime $\delta \geq \operatorname{poly}(1 / m)$ and $\Delta / \delta \geq \operatorname{poly}(m)$ which can be achieved if the chain is sufficiently close to the quantum phase transition point. The logical Pauli operator $\bar{X}_{u}$ will be simulated by the energy splitting between $\psi_{0}$ and $\psi_{1}$. The logical Pauli operator $\bar{Z}_{u}$ will be simulated by applying a magnetic field $h Z_{i}$ to an arbitrarily chosen qubit $i \in L_{u}$. The strength of the field $h$ will be much smaller than the gap $\Delta$ to enable a perturbative analysis. We shall only need the first-order perturbation theory. Since the one-dimensional TIM is exactly solvable, all parameters of the logical qubit will be efficiently computable. Assuming that the target model has $n$ qubits, the simulator model will be composed of $n$ chains $L_{1}, \ldots, L_{n}$ of length $m$ each. We can simulate a logical interaction $\bar{Z}_{u} \bar{Z}_{v}$ by choosing an arbitrary pair of qubits $i \in L_{u}, j \in L_{v}$ and applying the Ising interaction $Z_{i} Z_{j}$. Since each logical qubit $L_{u}$ is coupled to at most $n-1$ other logical qubits, choosing $m \geq n-1$ guarantees that each qubit of $L_{u}$ is coupled to at most one qubit from a different chain. In addition, each qubit of $L_{u}$ must be coupled to its left and right neighbors in $L_{u}$. Thus the simulator model has interactions of degree 3. A logical transverse field $\bar{X}_{u}$ is automatically simulated due to the ground state energy splitting of $L_{u}$. Thereby, we shall be able to simulate any logical TIM Hamiltonian.

Let us now describe the reduction formally. For the sake of clarity, we begin by constructing a single logical qubit. Consider a chain of $m$ qubits with periodic boundary conditions. Qubits will be labeled by elements of the cyclic group $j \in \mathbb{Z}_{m}$. Consider a TIM Hamiltonian

$$
H_{\text {chain }}=-g \sum_{j \in \mathbb{Z}_{m}} Z_{j} Z_{j+1}-\sum_{j \in \mathbb{Z}_{m}} X_{j}
$$

where

$$
g=1+\frac{c \log (m)}{m}
$$

for some parameter $c \gg 1$ to be chosen later. The Hamiltonian $H_{\text {chain }}$ can be diagonalized via the Jordan-Wigner transformation and all its eigenvalues have been explicitly computed [14]. Let the three smallest eigenvalues of $H_{\text {chain }}$ be $E_{0} \leq E_{1} \leq E_{2}$. We shall use the following well-known fact $[29,34]$.

Fact 1. Let $\omega_{m}=e^{2 \pi i / m}$ be the $m$-th root of unity. For any $g>1$ one has

$$
E_{0}=-\sum_{j \in \mathbb{Z}_{m}}\left|g-\omega_{m}^{j+1 / 2}\right|, \quad E_{1}=-\sum_{j \in \mathbb{Z}_{m}}\left|g-\omega_{m}^{j}\right|, \quad E_{2}=E_{0}+4\left|g-\omega_{m}^{1 / 2}\right| .
$$


Furthermore, the eigenvalues $E_{0}, E_{1}$ have multiplicity one and the corresponding eigenvectors $\psi_{0}, \psi_{1}$ satisfy $X^{\otimes m} \psi_{0}=\psi_{0}$ and $X^{\otimes m} \psi_{1}=-\psi_{1}$.

Let $\mathcal{H}=\left(\mathbb{C}^{2}\right)^{\otimes m}$ be the full Hilbert space of $n$ qubits. Define an encoding $\mathcal{E}: \mathbb{C}^{2} \rightarrow \mathcal{H}$ as

$$
\mathcal{E}|0\rangle \equiv|\overline{0}\rangle=\left(\left|\psi_{0}\right\rangle+\left|\psi_{1}\right\rangle\right) / \sqrt{2} \quad \text { and } \quad \mathcal{E}|1\rangle \equiv|\overline{1}\rangle=\left(\left|\psi_{0}\right\rangle-\left|\psi_{1}\right\rangle\right) / \sqrt{2}
$$

Thus we identify the states $\psi_{0}$ and $\psi_{1}$ with the states $|+\rangle$ and $|-\rangle$ of the logical qubit. Decompose $\mathcal{H}=\mathcal{H}_{-} \oplus \mathcal{H}_{+}$, where $\mathcal{H}_{-}$is the logical subspace spanned by $\psi_{0}, \psi_{1}$ and and $\mathcal{H}_{+}$is the orthogonal complement of $\mathcal{H}_{-}$. Then $H_{\text {chain }}$ is block-diagonal. Performing the overall energy shift by $\left(E_{0}+E_{1}\right) / 2$ we arrive at

$$
\left(H_{\text {chain }}\right)_{--}=-\delta \bar{X} \quad \text { and } \quad\left(H_{\text {chain }}\right)_{++} \geq \Delta I
$$

where $\bar{X}=\mathcal{E} X \mathcal{E}^{\dagger}=\left|\psi_{0}\right\rangle\left\langle\psi_{0}|-| \psi_{1}\right\rangle\left\langle\psi_{1}\right|$ is the logical Pauli $X$ operator,

$$
\delta=\left(E_{1}-E_{0}\right) / 2 \text { and } \Delta=4\left|g-\omega_{m}^{1 / 2}\right|-\delta .
$$

Note that $\delta$ and $\Delta$ can be computed in time poly $(m)$ using Eq. (38). In Appendix A we prove that

$$
\Omega\left(m^{-c-3 / 2}\right) \leq \delta \leq O\left(m^{-c-1 / 2}\right)
$$

in the limit $m \rightarrow \infty$. Therefore

$$
\Delta \geq \frac{4 c \log (m)}{m}-O\left(m^{-1}\right)-O\left(m^{-c-1 / 2}\right) \geq \Omega\left(m^{-1}\right) .
$$

By choosing the constant $c$ sufficiently large we can make the ratio $\Delta / \delta$ bigger than any fixed polynomial of $m$ and, at the same time, keep $\delta$ at least polynomial in $1 / \mathrm{m}$.

Consider now a perturbation $V=h Z_{j}$, where $|h| \ll \Delta$ and $j \in \mathbb{Z}_{m}$ is an arbitrarily chosen qubit. The first-order effective Hamiltonian acting on $\mathcal{H}_{-}$is $h\left(Z_{j}\right)_{--}$. To compute $\left(Z_{j}\right)_{--}$we need to know matrix elements $\left\langle\psi_{\alpha}\left|Z_{j}\right| \psi_{\beta}\right\rangle$ for $\alpha, \beta=0,1$. Note that $Z_{j}$ anti-commutes with $X^{\otimes m}$. Using Fact 1 we infer that $\left\langle\psi_{0}\left|Z_{j}\right| \psi_{0}\right\rangle=0$ and $\left\langle\psi_{1}\left|Z_{j}\right| \psi_{1}\right\rangle=0$. Therefore $\left(Z_{j}\right)_{--}$must be a linear combination of the logical Pauli operators $\bar{Z}$ and $\bar{Y}$. Since $H_{\text {chain }}$ has real matrix elements in the standard basis, the same is true for the restrictions of $H_{\text {chain }}$ onto the sectors $X^{\otimes m}= \pm 1$. Therefore $\psi_{0}$ and $\psi_{1}$ must have real amplitudes in the standard basis. This shows that $\left\langle\psi_{0}\left|Z_{j}\right| \psi_{1}\right\rangle$ must be real and thus

$$
\left(Z_{j}\right)_{--}=\xi \bar{Z}, \quad \text { where } \xi \equiv\left\langle\psi_{1}\left|Z_{j}\right| \psi_{0}\right\rangle
$$

and $\bar{Z}=\mathcal{E} Z \mathcal{E}^{\dagger}=\left|\psi_{0}\right\rangle\left\langle\psi_{1}|+| \psi_{1}\right\rangle\left\langle\psi_{0}\right|$ is the logical Pauli $Z$ operator. It can be easily shown that $\xi$ does not depend on the choice of $j$. We shall need the following expression for $\xi$ computed in Ref. [29], see Eq. (77) therein.

Fact 2. Suppose $g>1$. Let $\epsilon_{p} \equiv\left|g-\omega_{m}^{p}\right|$, where $p$ is either integer or half-integer. Then

$$
|\xi|=\frac{\left(1-g^{-2}\right)^{1 / 8} \prod_{p \in \mathbb{Z}_{m}} \prod_{q \in \mathbb{Z}_{m}+1 / 2}\left(\epsilon_{p}+\epsilon_{q}\right)^{1 / 4}}{\prod_{p, p^{\prime} \in \mathbb{Z}_{m}}\left(\epsilon_{p}+\epsilon_{p^{\prime}}\right)^{1 / 8} \prod_{q, q^{\prime} \in \mathbb{Z}_{m}+1 / 2}\left(\epsilon_{q}+\epsilon_{q^{\prime}}\right)^{1 / 8}}
$$


Furthermore, in Appendix A we prove that $\xi$ is positive and

$$
\xi \geq\left(1-g^{-2}\right)^{1 / 8} \geq \Omega\left(m^{-1 / 8}\right)
$$

for all $m \geq 2$ and for all $g>1$. Note that $\xi$ can be computed in time poly $(m)$ using Eq. (44). We can now simulate any target Hamiltonian on a single qubit which has a form

$$
H_{\text {target }}=-h^{x} X+h^{z} Z, \quad h^{x} \geq 0 .
$$

Let $J=\max \left(\left|h^{z}\right|, h^{x}\right)$ be the interaction strength of $H_{\text {target }}$. Choose the simulator Hamiltonian as

$$
H_{\text {sim }}=H_{0}+V, \quad H_{0}=h^{x} \delta^{-1} H_{\text {chain }}, \quad V=h^{z} \xi^{-1} Z_{j} .
$$

Here $j \in \mathbb{Z}_{m}$ is an arbitrary qubit. From Eqs. $(40,43)$ we infer that the first-order effective Hamiltonian acting on $\mathcal{H}_{-}$is

$$
H_{\text {eff }}(1)=\left(H_{\text {sim }}\right)_{--}=h^{x} \delta^{-1}\left(H_{\text {chain }}\right)_{--}+h^{z} \xi^{-1}\left(Z_{j}\right)_{--}=-h^{x} \bar{X}+h^{z} \bar{Z}=\bar{H}_{\text {target }} .
$$

Note that $H_{0}$ has an energy gap $\Delta^{\prime}=h^{x} \Delta \delta^{-1}$ separating $\mathcal{H}_{-}$and $\mathcal{H}_{+}$. By Lemma 7 , the Hamiltonian $H_{\text {sim }}$ and the encoding $\mathcal{E}$ simulate $H_{\text {target }}$ with an error $(\eta, \epsilon)$ provided that $\Delta^{\prime} \geq$ poly $\left(h^{z} \xi^{-1}, \epsilon^{-1}, \eta^{-1}\right)$ for some constant degree polynomial. We can assume without loss of generality that $h^{x} \geq \epsilon / 2$ since we only need to approximate the target Hamiltonian with an error $\epsilon / 2$, see Lemma 7 . Then $\Delta^{\prime} \geq \Omega\left(\epsilon m^{-1} \delta^{-1}\right)$. Here we used the bound $\Delta=\Omega\left(m^{-1}\right)$, see Eq. (42). Since $\left|h^{z}\right| \leq J$, we have to satisfy $\delta^{-1} \geq \operatorname{poly}\left(m, J, \xi^{-1}, \epsilon^{-1}, \eta^{-1}\right)$. Since $\xi^{-1}=O\left(m^{1 / 8}\right)$, see Eq. (45), this is equivalent to $\delta^{-1} \geq \operatorname{poly}\left(m, J, \epsilon^{-1}, \eta^{-1}\right)$. This can always be achieved by choosing a large enough constant $c$ in Eq. (37) since $\delta^{-1}=\Omega\left(m^{c+3 / 2}\right)$, see Eq. (41). Finally, we express $\delta^{-1}$ in terms of the interaction strength $J^{\prime}$ of the simulator Hamiltonian. From Eq. (47) one gets $J^{\prime}=O\left(h^{x} \delta^{-1}\right)$ and thus we can achieve a simulation error $(\eta, \epsilon)$ by choosing $J^{\prime}=\operatorname{poly}\left(m, J, \epsilon^{-1}, \eta^{-1}\right)$.

Consider now a target Hamiltonian on $n$ qubits which has a form

$$
H_{\text {target }}=\sum_{0 \leq u<v \leq n-1} \omega_{u, v} Z_{u} Z_{v}+\sum_{u=0}^{n-1} h_{u}^{z} Z_{u}-h_{u}^{x} X_{u}
$$

Without loss of generality $h_{u}^{x} \geq 0$ (otherwise, conjugate the Hamiltonian by $Z_{u}$ ). We shall encode each qubit $u$ into a chain $L_{u}$ of length $m=n$ as defined above. Let $H_{\text {chain }}^{(u)}$ be the Hamiltonian Eq. (36) describing the chain $L_{u}$. We shall arrange the chains into a square grid of size $n \times n$ such that a cell $(u, i)$ of the grid represents the $i$-th qubit of the chain $L_{u}$. Here $0 \leq u, i \leq n-1$. All chains use the same parameter $g$. Choose the simulator Hamiltonian as

$$
H_{\text {sim }}=H_{0}+V, \quad H_{0}=\delta^{-1} \sum_{u=0}^{n-1} h_{u}^{x} H_{\text {chain }}^{(u)}
$$




$$
V=\xi^{-2} \sum_{0 \leq u<v \leq n-1} \omega_{u, v} Z_{(u, v)} Z_{(v, u)}+\xi^{-1} \sum_{u=0}^{n-1} h_{u}^{z} Z_{(u, 0)} .
$$

Note that $H_{\text {sim }}$ is a TIM Hamiltonian acting on $n^{2}$ qubits and such that each qubit is coupled to at most three other qubits with $Z Z$ interactions. Namely, a qubit $(u, v)$ is coupled only to the qubits $(u, v \pm 1)$ and $(v, u)$. Let $\mathcal{H}_{-}$be the $n$-fold tensor product of the two-dimensional logical subspaces describing each chain $L_{u}$. The above analysis for a single logical qubit shows that $\left(H_{\text {chain }}^{(u)}\right)_{--}=-\delta \bar{X}_{u}$ and $\left(Z_{(u, v)}\right)_{--}=\xi \bar{Z}_{u}$ for any qubit $v$ in the chain $L_{u}$. Therefore the first-order effective Hamiltonian acting on $\mathcal{H}_{-}$is

$$
H_{\mathrm{eff}}(1)=\left(H_{\mathrm{sim}}\right)_{--}=\sum_{0 \leq u<v \leq n-1} \omega_{u, v} \bar{Z}_{u} \bar{Z}_{v}+\sum_{u=0}^{n-1} h_{u}^{z} \bar{Z}_{u}-h_{u}^{x} \bar{X}_{u}=\bar{H}_{\text {target }},
$$

where the encoding $\mathcal{E}$ is the $n$-fold tensor product of single qubit encodings defined in Eq. (39).

Let $(\eta, \epsilon)$ be the desired simulation error and $J$ be the maximum magnitude of the coefficients in $H_{\text {target }}$. We can assume without loss of generality that $h_{u}^{x} \geq \epsilon / 2 n$ for all $u$. Then the energy gap of $H_{0}$ separating $\mathcal{H}_{-}$from excited states is $\Delta^{\prime} \geq \epsilon \delta^{-1} \Delta / 2 n$, where $\Delta$ is the energy gap of a single chain, see Eq. (40). By Lemma 7, the Hamiltonian $H_{\text {sim }}$ and the encoding $\mathcal{E}$ simulate $H_{\text {target }}$ with an error $(\eta, \epsilon)$ provided that $\Delta^{\prime} \geq \operatorname{poly}\left(n, J, \epsilon^{-1}, \eta^{-1}\right)$. This is equivalent to $\delta^{-1} \geq \operatorname{poly}\left(n, J, \epsilon^{-1}, \eta^{-1}\right.$ ) (use the same bounds as above), which can always be satisfied by choosing a large enough constant $c$ in Eq. (37). Then the simulator Hamiltonian has interaction strength $J^{\prime}=O\left(\delta^{-1} J\right)=\operatorname{poly}\left(n, J, \epsilon^{-1}, \eta^{-1}\right)$.

To conclude, we have shown that any Hamiltonian $H_{\text {target }} \in \operatorname{TIM}(n, J)$ can be simulated with an error $(\eta, \epsilon)$ by a Hamiltonian $H_{\text {sim }} \in \operatorname{TIM}\left(n^{2}, J^{\prime}\right)$ such that $J^{\prime}=\operatorname{poly}\left(n, J, \epsilon^{-1}, \eta^{-1}\right)$ and $H_{\text {sim }}$ has interaction degree 3. The simulation uses the encoding $\mathcal{E}$ defined in Eq. (39). Furthermore, the coefficients of $H_{\text {sim }}$ can be computed in time poly $(n)$.

\section{Reduction from TIM to dimers}

In this section we construct a TIM simulator for the hard-core dimers model. It involves a composition of a first-order and a second-order reduction. First let us construct a classical Ising Hamiltonian $H_{0}$ composed of terms proportional to $n_{u}$ and $n_{u} n_{v}$ such that ground states of $H_{0}$ are $m$-dimers. Consider a graph $G=(U, E)$ with $n$ nodes. Define operators

$$
N_{U}=\sum_{u \in U} n_{u} \text { and } N_{E}=\sum_{(u, v) \in E} n_{u} n_{v}
$$

These operators act on the full Hilbert space $\mathcal{B} \cong\left(\mathbb{C}^{2}\right)^{\otimes n}$. Define

$$
H_{0}=N_{U}-2 N_{E}+\Gamma \sum_{D(u, v)=2} n_{u} n_{v}, \quad \Gamma>2|E| .
$$

Here $D(u, v)$ denotes the graph distance between nodes $u, v$. Note that $H_{0}$ is a TIM Hamiltonian (with a zero transverse field). 
Lemma 8. The Hamiltonian $H_{0}$ has zero ground state energy and its ground subspace is spanned by $m$-dimers with $0 \leq m \leq n / 2$. Furthermore, if $S$ is an $m$-dimer and $T=S \backslash u$ for some $u \in S$ then $\left\langle T\left|H_{0}\right| T\right\rangle=1$.

Proof. Suppose $S \subseteq U$ is an $m$-dimer. By definition, any pair of dimers in $S$ is separated by distance at least three. Thus $\left\langle S\left|n_{u} n_{v}\right| S\right\rangle=0$ whenever $D(u, v)=2$. Therefore

$$
\left\langle S\left|H_{0}\right| S\right\rangle=\left\langle S\left|N_{U}\right| S\right\rangle-2\left\langle S\left|N_{E}\right| S\right\rangle=2 m-2 m=0 .
$$

Next consider any subset of nodes $S$ such that $\left\langle S\left|H_{0}\right| S\right\rangle \leq 0$. It suffices to show that $S$ is an $m$-dimer for some integer $m$. Indeed, the negative term in $H_{0}$ cannot be smaller than $-2|E|$. Since $\Gamma>2|E|$, the energy of $S$ can be non-positive only if

$$
\left\langle S\left|n_{u} n_{v}\right| S\right\rangle=0 \quad \text { whenever } D(u, v)=2 .
$$

Let $S=C_{1} \cup \ldots \cup C_{m}$ be the decomposition of $S$ into connected components. Since the graph has no triangles, Eq. (54) implies that each connected component of $S$ is either a single node or a dimer. Therefore

$$
0 \geq\left\langle S\left|H_{0}\right| S\right\rangle=\sum_{\alpha=1}^{m}\left\langle C_{\alpha}\left|N_{U}-2 N_{E}\right| C_{\alpha}\right\rangle .
$$

Clearly, $\left\langle C_{\alpha}\left|N_{U}-2 N_{E}\right| C_{\alpha}\right\rangle=1$ if $C_{\alpha}$ is a single node and $\left\langle C_{\alpha}\left|N_{U}-2 N_{E}\right| C_{\alpha}\right\rangle=0$ if $C_{\alpha}$ is a dimer. Thus Eq. (55) is possible only if all $C_{\alpha}$ are dimers. From Eq. (54) one infers that the distance between different dimers $C_{\alpha}$ is at least three. This shows that $S$ is an $m$-dimer for some $m$.

Finally, removing any single node $u$ from an $m$-dimer $S$ transforms one of the connected components $C_{\alpha}$ into a single node. The above shows that $T=S \backslash u$ has energy $\left\langle T\left|H_{0}\right| T\right\rangle=1$.

Fix any integer $1 \leq m \leq n / 2$. Consider a target Hamiltonian $H_{\text {target }} \in \operatorname{HCD}(n, m, J)$ describing the $m$-dimer sector of the hard-core dimers model on some triangle-free graph $G=(U, E)$ with $n$ nodes, see Eq. (5).

Our first reduction has a simulator Hamiltonian

$$
\tilde{H}_{\text {sim }}=\tilde{\Delta} \tilde{H}_{0}+\tilde{V}, \quad \tilde{H}_{0}=\left(N_{U}-2 m\right)\left(N_{U}-2 m+1\right), \quad \tilde{V} \in \operatorname{TIM}(n, \tilde{J}) .
$$

All above operators act on the full Hilbert space $\mathcal{B}$. The perturbation $\tilde{V}$ will be chosen at the next reduction. Note that $\tilde{H}_{\text {sim }}$ is a TIM Hamiltonian. Since eigenvalues of $N_{U}$ are integers, the ground subspace of $\tilde{H}_{0}$ is spanned by subsets of nodes of cardinality $2 m$ or $2 m-1$. By Lemma 4 , the Hamiltonian $\tilde{H}_{\text {sim }}$ can simulate the restriction of any TIM Hamiltonian $\tilde{V}$ onto the subspace $\mathcal{H} \equiv \mathcal{B}_{2 m} \oplus \mathcal{B}_{2 m-1}$. In the rest of this section we assume that our full Hilbert space is $\mathcal{H}$.

Our second reduction has a simulator Hamiltonian

$$
H_{\text {sim }}=\Delta H_{0}+V, \quad V=\Delta^{1 / 2} V_{\text {main }}+V_{\text {extra }},
$$


where $H_{0}$ is the Hamiltonian constructed in Lemma 8,

$$
V_{\text {main }}=t^{1 / 2} \sum_{u \in U} X_{u} \quad \text { and } \quad V_{\text {extra }}=H_{\text {diag }}+t N_{U}
$$

Here $t$ and $H_{\text {diag }}$ are defined by the target HCD Hamiltonian Eq. (5) and all operators are restricted to the subspace $\mathcal{H}$ with $2 m$ or $2 m-1$ particles. Note that $H_{\text {sim }}$ is a TIM Hamiltonian. Lemma 8 implies that the ground subspace of $H_{0}$ is spanned by $m$-dimers, that is, $\mathcal{H}_{-}=\mathcal{D}_{m}$.

Let us check that the perturbation has all the properties stated in Lemma 5. First, we note that $\left(V_{\text {main }}\right)_{--}=0$ since any pair of $m$-dimers either coincide or differ on at least two nodes. Obviously, $V_{\text {extra }}$ is block-diagonal. It remains to check Eq. (32). Let $S$ and $S^{\prime}$ be arbitrary $m$-dimers. Then

$$
\left\langle S^{\prime}\left|\left(V_{\text {main }}\right)_{-+} H_{0}^{-1}\left(V_{\text {main }}\right)_{+-}\right| S\right\rangle=t \sum_{u, v \in U}\left\langle S^{\prime}\left|X_{v} P_{+} H_{0}^{-1} P_{+} X_{u}\right| S\right\rangle .
$$

Recall that all operators in Eq. (57) are restricted to the subspace with $2 m$ or $2 m-1$ particles. Thus $X_{u}|S\rangle=0$ whenever $u \notin S$ since in this case $X_{u}|S\rangle$ contains $2 m+1$ particles. In the remaining case, $u \in S$, Lemma 8 implies that $X_{u}|S\rangle$ is an eigenvector of $H_{0}$ with the eigenvalue 1 , so that $H_{0}^{-1} X_{u}|S\rangle=X_{u}|S\rangle$. Thus

$$
\left\langle S^{\prime}\left|\left(V_{\text {main }}\right)_{-+} H_{0}^{-1}\left(V_{\text {main }}\right)_{+-}\right| S\right\rangle=t \sum_{u \in S, v \in S^{\prime}}\left\langle S^{\prime}\left|X_{v} X_{u}\right| S\right\rangle
$$

The sum over $u=v$ gives a contribution $t|S| \delta_{S, S^{\prime}}=\left\langle S\left|t N_{U}\right| S^{\prime}\right\rangle$. The sum over $u \neq v$ is non-zero only if $S$ and $S^{\prime}$ can be obtained from each other by moving one particle from some node $u$ to another node $v$, in which case $\left\langle S^{\prime}\left|X_{v} X_{u}\right| S\right\rangle=\left\langle S^{\prime}\left|W_{u, v}\right| S\right\rangle$. Thus

$$
\left(V_{\text {main }}\right)_{-+} H_{0}^{-1}\left(V_{\text {main }}\right)_{+-}=t N_{U}+t \sum_{\{u, v\} \in U} W_{u, v}
$$

and

$$
\left(V_{\text {extra }}\right)_{--}-\left(V_{\text {main }}\right)_{-+} H_{0}^{-1}\left(V_{\text {main }}\right)_{+-}=-t \sum_{\{u, v\} \in U} W_{u, v}+H_{\text {diag }}=H_{\text {target }} .
$$

Thus all conditions of Lemma 5 are satisfied.

To compose the two reductions we extend $H_{\text {sim }}$ defined in Eq. (57) to the full Hilbert space $\mathcal{B}$ and substitute $\tilde{V}=H_{\text {sim }}$ into Eq. (56). Combining Lemmas 3,4,5 we conclude that any Hamiltonian $H_{\text {target }} \in \operatorname{HCD}(n, m, J)$ can be simulated with an error $(\epsilon, \eta)$ by a Hamiltonian $\tilde{H}_{\text {sim }} \in \operatorname{TIM}\left(n, J^{\prime}\right)$ where $J^{\prime}=\operatorname{poly}\left(n, J, \epsilon^{-1}, \eta^{-1}\right)$. The simulation uses the trivial encoding $\mathcal{E}: \mathcal{D}_{m} \rightarrow \mathcal{B}$, that is, $\mathcal{E}|S\rangle=|S\rangle$ for any $m$-dimer $S$. 


\section{Reduction from dimers to range-2 bosons}

In this section we construct an HCD simulator for range-2 hard-core bosons. It involves a third-order reduction. Consider a target Hamiltonian $H_{\text {target }} \in \mathrm{HCB}_{2}(n, m, J)$ describing the $m$-particle sector of range- 2 hard-core bosons on some graph $G=(U, E)$ with $n$ nodes. For the sake of clarity, let us first consider a special case of homogeneous hopping amplitudes, that is,

$$
H_{\text {target }}=-t \sum_{(u, v) \in E} W_{u, v}+H_{\text {diag }}, \quad t \geq 0 .
$$

Recall that $H_{\text {target }}$ acts on the Hilbert space $\mathcal{B}_{m, 2}(G)$ spanned by 2 -sparse subsets of $m$ nodes.

The HCD simulator will be defined on an extended graph $G^{\prime}=\left(U^{\prime}, E^{\prime}\right)$ obtained from $G$ by placing an extra node at the center of every edge of $G$ and attaching an extra hanging edge to every node of $G$, see Fig. 2 for an example. The extra node located at the center of an edge $(u, v) \in E$ will be denoted ${ }^{3} u+v$. The extra node attached to a node $u \in U$ by a hanging edge will be denoted $u^{*}$. Thus the extended graph $G^{\prime}$ has a set of nodes

$$
U^{\prime}=U \cup U^{*} \cup U^{+}, \quad U^{*}=\left\{u^{*}: u \in U\right\}, \quad U^{+}=\{u+v: u, v \in U \text { and }(u, v) \in E\} .
$$

We shall represent a boson located at a node $u \in U$ by a dimer occupying the subset $\left\{u, u^{*}\right\} \subseteq U^{\prime}$.
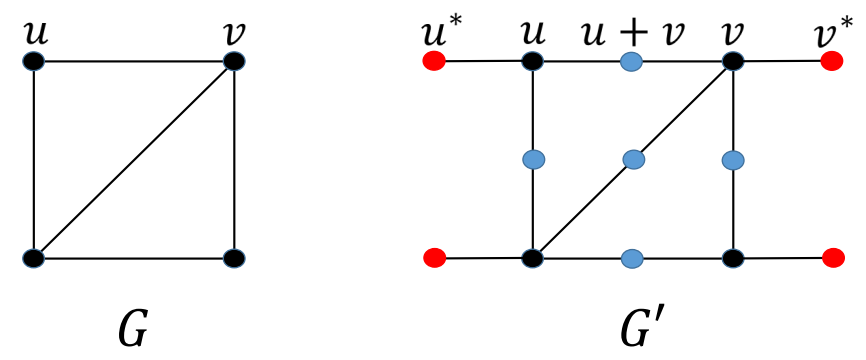

Figure 2: Construction of the extended graph $G^{\prime}=\left(U^{\prime}, E^{\prime}\right)$ for hard-core dimers starting from the graph $G=(U, E)$ of the hard-core bosons model. The subsets of nodes $U^{*}$ and $U^{+}$are highlighted in red and blue respectively. A boson located at a node $u \in U$ is represented by a dimer occupying the subset $\left\{u, u^{*}\right\} \subseteq U^{\prime}$.

A simulator HCD Hamiltonian acting on the Hilbert space of $m$-dimers $\mathcal{D}_{m}\left(G^{\prime}\right)$ is defined as $H_{\text {sim }}=\Delta H_{0}+V$, where

$$
H_{0}=\sum_{w \in U^{+}} \Delta_{w} n_{w}
$$

penalizes $m$-dimers occupying the extra nodes located at the centers of edges of $G$. For now we set $\Delta_{w}=1$ for all $w \in U^{+}$. We shall need a more general expression for $\Delta_{w}$ in the case of nonhomogeneous hopping amplitudes. Clearly, $H_{0}$ has zero ground state energy and its ground subspace

\footnotetext{
${ }^{3}$ Here the addition is merely a symbol; it has no algebraic meaning.
} 
is spanned by $m$-dimers $S \subseteq U^{\prime}$ such that $S \cap U^{+}=\emptyset$. The perturbation is defined as $V=$ $\Delta^{2 / 3} V_{\text {main }}+\Delta^{1 / 3} \tilde{V}_{\text {extra }}+V_{\text {extra }}$, where

$$
\begin{gathered}
V_{\text {main }}=-t^{1 / 3} \sum_{\{u, v\} \in U^{\prime}} W_{u, v}, \\
\tilde{V}_{\text {extra }}=t^{2 / 3} \sum_{u \in U} d(u) n_{u} . \\
V_{\text {extra }}=H_{\text {diag }}+t \sum_{u \in U} d_{2}(u) n_{u},
\end{gathered}
$$

For now we define $d(u)$ as the degree of a node $u$ in the original graph $G$ and $d_{2}(u) \equiv d(u)(d(u)-1)$. We shall need a more general expression for $d(u)$ and $d_{2}(u)$ in the case of non-homogeneous hopping amplitudes.

Let us check that the perturbation has all the properties stated in Lemma 6. First, we claim that $\left(V_{\text {main }}\right)_{--}=0$. Indeed, suppose $|S\rangle$ is a ground state of $H_{0}$. Then $S$ must be a union of dimers $\left\{u, u^{*}\right\}$ with $u \in U$. Since $\left(u, u^{*}\right)$ is the only edge of $G^{\prime}$ attached to $u^{*}$, the only hopping terms that can map $S$ to some $m$-dimer $S^{\prime}$ are those that replace some dimer $\left\{u, u^{*}\right\} \subseteq S$ with a dimer $\{u, u+v\}$ for some $(u, v) \in E$, see Fig. 3. This requires a single hopping from $u^{*}$ to $u+v$. Then $S^{\prime}$ has a particle at some node $u+v$ and thus $\left|S^{\prime}\right\rangle$ is an excited state of $H_{0}$. Thus $\left(V_{\text {main }}\right)_{--}=0$. The operators $V_{\text {extra }}$ and $\tilde{V}_{\text {extra }}$ are block-diagonal simply because they are diagonal.

Let us now describe the encoding $\mathcal{E}: \mathcal{B}_{m, 2}(G) \rightarrow \mathcal{D}_{m}\left(G^{\prime}\right)$. Recall that $\mathcal{B}_{m, 2}(G)$ and $\mathcal{D}_{m}\left(G^{\prime}\right)$ are the Hilbert spaces of the target and the simulator models. Given a 2-sparse subset of nodes $S \subseteq U$ in the graph $G$ let $\mathcal{E}(S) \subseteq U \cup U^{*}$ be the subset of nodes in the graph $G^{\prime}$ that includes all nodes $u \in S$ and all nodes $u^{*}$ such that $u \in S$. Define $\mathcal{E}|S\rangle=|\mathcal{E}(S)\rangle$. Obviously, $\mathcal{E}$ is an isometry. Let us check that $\operatorname{Im}(\mathcal{E})$ coincides with the ground subspace of $H_{0}$. Indeed, suppose $S \subseteq U^{\prime}$ is a ground state of $H_{0}$, that is, $S$ is an $m$-dimer in $G^{\prime}$ such that $S \subseteq U \cup U^{*}$. Then all dimers in $S$ must have a form $\left\{u, u^{*}\right\}$ for some $u \in U$. Consider any distinct nodes $u, v \in S \cap U$. By definition of an $m$-dimer, any dimers in $S$ are separated by at least three edges in the graph $G^{\prime}$. Then the nodes $u$ and $v$ are separated by at least two edges in the graph $G$, that is, $S \cap U$ is a 2-sparse subset of $m$ nodes in the graph $G$. Since $S=\mathcal{E}(S \cap U)$, this shows that $S$ belongs to the image of $\mathcal{E}$. Conversely, if $S \subseteq U$ is any 2-sparse subset of $m$ nodes in $G$ then $\mathcal{E}(S)$ is an $m$-dimer in $G$ such that $\mathcal{E}(S) \subseteq U \cup U^{*}$. This proves that $\operatorname{Im}(\mathcal{E})$ coincides with the ground subspace of $H_{0}$.

Let us now check condition Eq. (33) of Lemma 6. Consider any m-dimer $S \subseteq U^{\prime}$ such that $S \cap U^{+}=\emptyset$. We have already shown that $V_{\text {main }}|S\rangle$ is a superposition of states $\left|S^{\prime}\right\rangle$, where $S^{\prime}$ is obtained from $S$ by replacing a dimer $\left\{u, u^{*}\right\}$ with a dimer $\{u, u+v\}$ for some $u \in S \cap U$ and $v \in U$ such that $(u, v) \in E$, see Fig. 3. By definition of an $m$-dimer, $\left\{u, u^{*}\right\}$ is separated from all other dimers of $S$ by at least three edges of the graph $G^{\prime}$. However, since $S \cap U^{+}=\emptyset$, this is possible only if $\left\{u, u^{*}\right\}$ is separated from all other dimers of $S$ by at least four edges of $G^{\prime}$. Then the dimer $\{u, u+v\}$ is separated from all other dimers of $S^{\prime}$ by at least three edges of $G^{\prime}$, that is, $S^{\prime}$ is an $m$-dimer. Note also that $S^{\prime}$ occupies exactly one node of $U^{+}$, that is, $H_{0}\left|S^{\prime}\right\rangle=\left|S^{\prime}\right\rangle$ and thus $H_{0}^{-1}\left|S^{\prime}\right\rangle=\left|S^{\prime}\right\rangle$ (recall 
that we set $\Delta_{w}=1$ in the case of homogeneous hopping amplitudes). The above arguments show that

$$
\left(V_{\text {main }}\right)_{+-}|S\rangle=-t^{1 / 3} \sum_{u \in S \cap U} \sum_{v:(u, v) \in E} W_{u^{*}, u+v}|S\rangle
$$

Using the above equation one can easily get

$$
\begin{aligned}
\left\langle S^{\prime}\left|\left(V_{\text {main }}\right)_{-+} H_{0}^{-1}\left(V_{\text {main }}\right)_{++} H_{0}^{-1}\left(V_{\text {main }}\right)_{+-}\right| S\right\rangle= & -t \sum_{(u, v) \in E}\left\langle S^{\prime}\left|W_{u+v, v^{*}} W_{u, v} W_{u^{*}, u+v}\right| S\right\rangle \\
& -t \sum_{(u, v) \neq(u, w) \in E}\left\langle S^{\prime}\left|W_{u+w, u^{*}} W_{u+v, u+w} W_{u^{*}, u+v}\right| S\right\rangle
\end{aligned}
$$

Here $S^{\prime}$ is some $m$-dimer $S^{\prime} \subseteq U^{\prime}$ such that $S^{\prime} \cap U^{+}=\emptyset$. The terms in the first and the second line in the righthand side of Eq. (70) describe triple-hopping processes shown on Fig. 3 and Fig. 4 respectively. The former implements a logical hopping operator $\bar{W}_{u, v}=\mathcal{E} W_{u, v} \mathcal{E}^{\dagger}$, while the latter generates unwanted terms proportional to $\bar{n}_{u} d(u)(d(u)-1)$, where $d(u)$ is the degree of $u$ in the graph $G$. Thus

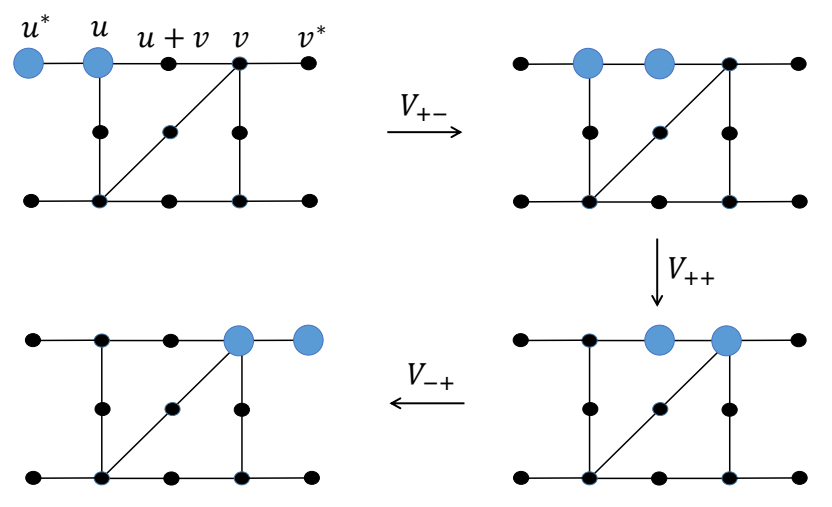

Figure 3: A third-order process transfers a dimer from $\left\{u, u^{*}\right\}$ to $\left\{v, v^{*}\right\}$. Here $V \equiv V_{\text {main }}$. Since each dimer in $G^{\prime}$ encodes one particle in $G$, this process simulates the logical hopping operator $\bar{W}_{u, v}$.

$$
\left(V_{\text {main }}\right)_{-+} H_{0}^{-1}\left(V_{\text {main }}\right)_{++} H_{0}^{-1}\left(V_{\text {main }}\right)_{+-}=-t \sum_{(u, v) \in E} \bar{W}_{u, v}-t \sum_{u \in U} d_{2}(u) \bar{n}_{u} .
$$

Note that the last term is canceled by $\left(V_{\text {extra }}\right)_{--}$, so that

$$
\left(V_{\text {extra }}\right)_{--}+\left(V_{\text {main }}\right)_{-+} H_{0}^{-1}\left(V_{\text {main }}\right)_{++} H_{0}^{-1}\left(V_{\text {main }}\right)_{+-}=\bar{H}_{\text {diag }}-t \sum_{(u, v) \in E} \bar{W}_{u, v}=\bar{H}_{\text {target }}
$$




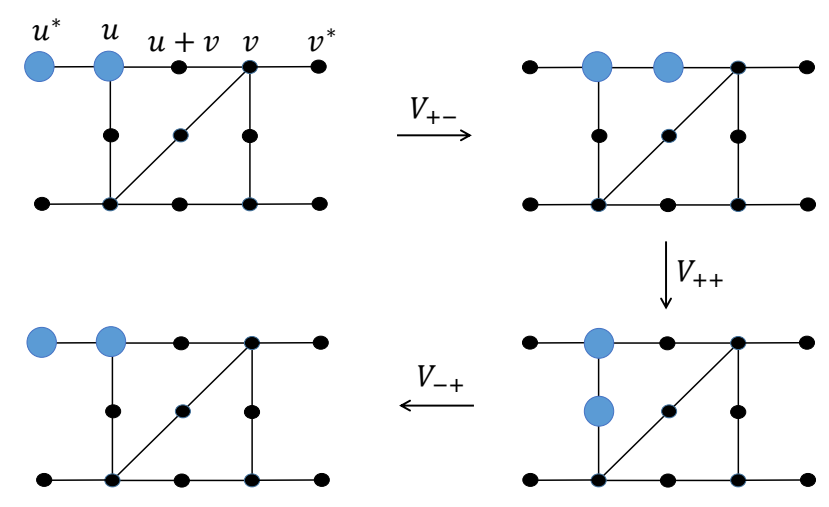

Figure 4: An unwanted third-order process transfers a dimer from $\left\{u, u^{*}\right\}$ back to $\left\{u, u^{*}\right\}$.

which proves condition Eq. (33) of Lemma 6. It remains to check condition Eq. (34). Using Eq. (69) again one gets

$$
\left\langle S^{\prime}\left|\left(V_{\text {main }}\right)_{-+} H_{0}^{-1}\left(V_{\text {main }}\right)_{+-}\right| S\right\rangle=t^{2 / 3} \sum_{u \in S} d(u) \delta_{S, S^{\prime}}=\left\langle S^{\prime}\left|t^{2 / 3} \sum_{u \in U} d(u) n_{u}\right| S\right\rangle=\left\langle S^{\prime}\left|\tilde{V}_{\text {extra }}\right| S\right\rangle .
$$

Here $\left(V_{\text {main }}\right)_{+-}$moves a particle from $u^{*}$ to $u+v$ and $\left(V_{\text {main }}\right)_{-+}$returns the particle back from $u+v$ to $u^{*}$. Thus all conditions of Lemma 6 are satisfied.

Suppose now that $H_{\text {target }} \in \mathrm{HCB}_{2}(n, m, J)$ has non-homogeneous hopping amplitudes, that is,

$$
H_{\text {target }}=-\sum_{(u, v) \in E} t_{u, v} W_{u, v}+H_{\text {diag }}, \quad 0 \leq t_{u, v} \leq t
$$

By definition, $t \leq J$. Let $(\eta, \epsilon)$ be the desired simulation error, see Definition 1. Since we only need to approximate $H_{\text {target }}$ with an error $\epsilon / 2$, see Lemma 6 , we can assume that

$$
\frac{\epsilon}{2|E|} \leq t_{u, v} \leq t \quad \text { for all }(u, v) \in E .
$$

For each node $w=u+v \in U^{+}$define

$$
\Delta_{w}=\sqrt{\frac{t}{t_{u, v}}} .
$$

Note that $1 \leq \Delta_{w} \leq \sqrt{2 J \epsilon^{-1}|E|} \leq \operatorname{poly}\left(n, J, \epsilon^{-1}\right)$. Given a node $u \in U$, let $\mathcal{N}(u) \subseteq U$ be the set of all nearest neighbors of $u$ in the graph $G$. Define

$$
d(u)=t^{-1 / 2} \sum_{v \in \mathcal{N}(u)} \sqrt{t_{u, v}} \quad \text { and } \quad d_{2}(u)=t^{-1} \sum_{v \neq v^{\prime} \in \mathcal{N}(u)} \sqrt{t_{u, v} t_{u, v^{\prime}}}
$$

Let $H_{\text {sim }}$ be the HCD simulator defined by Eqs. (65-68). Exactly the same arguments as above show that $H_{\text {sim }}$ satisfies conditions of Lemma 6 with the target Hamiltonian Eq. (73). 
We conclude that any Hamiltonian $H_{\text {target }} \in \mathrm{HCB}_{2}(n, m, J)$ can be simulated with an error $(\eta, \epsilon)$ by a Hamiltonian $H_{\text {sim }} \in \operatorname{HCD}\left(n^{\prime}, m, J^{\prime}\right)$ where $n^{\prime}=O\left(n^{2}\right)$ and $J^{\prime}=\operatorname{poly}\left(n, J, \epsilon^{-1}, \eta^{-1}\right)$. The simulation uses an encoding $\mathcal{E}$ that represents each particle of the target model by a dimer in the simulator model. In particular, $\mathcal{E}$ maps basis vectors to basis vectors. Note that the extended graph $G^{\prime}$ is triangle-free regardless of the original graph $G$, so the reduction from TIM to HCD described in Section 6 and the reduction from HCD to range-2 HCB can be composed.

\section{Range-2 bosons with multi-particle interactions}

In this section we describe a second-order reduction that uses range-2 $\mathrm{HCB}$ as a simulator and generates the same range-2 HCB Hamiltonian but with certain additional multi-particle interactions. This reduction is only needed for the proof of Theorem 2 .

Consider a graph $G=(U, E)$. For any subset of nodes $S \subseteq U$ define a diagonal operator

$$
D(S)=\prod_{u \in S}\left(I-n_{u}\right) .
$$

Let $d \leq \operatorname{poly}(n)$ be any integer and $S_{1}, \ldots, S_{d} \subseteq U$ be arbitrary subsets of nodes. Suppose our target Hamiltonian is

$$
H_{\text {target }}=H_{b o s}-\sum_{\alpha=1}^{d} p_{\alpha} D\left(S_{\alpha}\right) .
$$

Here $H_{b o s} \in \mathrm{HCB}_{2}(n, m, J)$ describes the range-2 $\mathrm{HCB}$ on the graph $G$ and $0 \leq p_{\alpha} \leq J$ are arbitrary coefficients. The Hamiltonian $H_{\text {target }}$ acts on the $m$-particle sector $\mathcal{B}_{m}(G)$. Let us show how to simulate $H_{\text {target }}$ using the standard range- $2 \mathrm{HCB}$ model. The simulator will be defined on an extended graph $G^{\prime}=\left(U^{\prime}, E^{\prime}\right)$ obtained from $G$ by adding extra nodes and extra edges. For each interaction $D\left(S_{\alpha}\right)$ in Eq. (74) let us add two extra nodes denoted $a(\alpha)$ and $b(\alpha)$. We connect the node $b(\alpha)$ by an edge with every node $u \in S_{\alpha}$. In addition, we connect the nodes $a(\alpha)$ and $b(\alpha)$ with each other, see Fig. 5. The number of particles in the simulator model is $m^{\prime}=m+d$, where $d$ is the number of extra terms in Eq. (74). Define a simulator Hamiltonian as

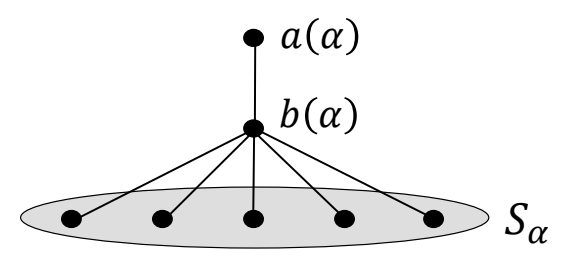

Figure 5: Simulation of multi-particle interactions $D\left(S_{\alpha}\right)$. We choose $H_{0}=I-n_{a(\alpha)}$ such that the node $a(\alpha)$ is occupied for any ground state of $H_{0}$. Then the node $b(\alpha)$ must be empty due to the 2 -sparsity constraint. The perturbation $V$ moves the particle from $a(\alpha)$ to $b(\alpha)$ or vice verse. 


$$
\begin{gathered}
H_{\text {sim }}=\Delta H_{0}+V, \quad H_{0}=\sum_{\alpha=1}^{d} I-n_{a(\alpha)}, \quad V=\Delta^{1 / 2} V_{\text {main }}+V_{\text {extra }}, \\
V_{\text {main }}=-\sum_{\alpha=1}^{d} \sqrt{p_{\alpha}} W_{a(\alpha), b(\alpha)} \quad \text { and } \quad V_{\text {extra }}=H_{b o s} .
\end{gathered}
$$

Clearly, $H_{0}$ has zero ground state energy and the ground subspace of $H_{0}$ is spanned by all 2-sparse configurations of particles in $G^{\prime}$ such that the node $a(\alpha)$ is occupied for each $\alpha$. Note that the node $b(\alpha)$ must be empty due to the 2 -sparsity constraint. Define an encoding $\mathcal{E}: \mathcal{B}_{m}(G) \rightarrow \mathcal{B}_{m+d}\left(G^{\prime}\right)$ as follows. If $S \subseteq U$ is a 2-sparse subset, define $\mathcal{E}(S)=S \cup\{a(1), \cdots, a(d)\} \subseteq U^{\prime}$. Note that $\mathcal{E}(S)$ is a 2-sparse subset since a node $a(\alpha)$ has only one neighbor $b(\alpha)$ and the latter never belongs to $\mathcal{E}(S)$. Define $\mathcal{E}|S\rangle=|\mathcal{E}(S)\rangle$. The above shows that $\mathcal{E}$ is an isometry and the image of $\mathcal{E}$ coincides with the ground subspace of $H_{0}$. Let us check that the perturbation $V$ satisfies conditions of Lemma 5 . Obviously, $\left(V_{\text {main }}\right)_{--}=0$ since any term in $V_{\text {main }}$ moves a particle from $a(\alpha)$ to $b(\alpha)$ or vice verse. The operator $V_{\text {extra }}$ is block-diagonal since it acts trivially on the extra nodes $a(\alpha), b(\alpha)$. Let us check condition Eq. (32) of Lemma 5. Note that no hopping in the original graph $G$ is prohibited due to the presence of extra particles at $a(\alpha)$ since these particles are separated from any node of $G$ by at least two edges. Thus $\left(V_{\text {extra }}\right)_{--}=\bar{H}_{b o s}$, where $\bar{H}_{b o s}=\mathcal{E} H_{b o s} \mathcal{E}^{\dagger}$ is the encoded version of $H_{b o s}$. Let us compute $\left(V_{\text {main }}\right)_{-+} H_{0}^{-1}\left(V_{\text {main }}\right)_{+-}$. Suppose $|S\rangle$ is a ground state of $H_{0}$. The 2 -sparsity condition implies that a node $b(\alpha)$ cannot be occupied if $S_{\alpha}$ contains at least one particle. This shows that $\left(W_{a(\alpha), b(\alpha)}\right)_{+-}|S\rangle=0$ if $S \cap S_{\alpha} \neq \emptyset$, Otherwise, $\left(W_{a(\alpha), b(\alpha)}\right)_{+-}$moves the particle from $a(\alpha)$ to $b(\alpha)$. Thus

$$
\left(W_{a(\alpha), b(\alpha)}\right)_{+-}|S\rangle=D\left(S_{\alpha}\right)|(S \backslash a(\alpha)) \cup b(\alpha)\rangle .
$$

Note that the state in the righthand side is an eigenvector of $H_{0}$ with an eigenvalue 1 . Note also that if $\left(V_{\text {main }}\right)_{+-}$moves a particle from some node $a(\alpha)$ to $b(\alpha)$ then $\left(V_{\text {main }}\right)_{-+}$must return the particle from $b(\alpha)$ to $a(\alpha)$. Thus

$$
\left(V_{\text {main }}\right)_{-+} H_{0}^{-1}\left(V_{\text {main }}\right)_{+-}=\sum_{\alpha=1}^{r} p_{\alpha}\left(W_{a(\alpha), b(\alpha)}\right)_{-+}\left(W_{a(\alpha), b(\alpha)}\right)_{+-}=\sum_{\alpha=1}^{r} p_{\alpha} \overline{D\left(S_{\alpha}\right)}
$$

Here we noted that $D\left(S_{\alpha}\right)^{2}=D\left(S_{\alpha}\right)=\overline{D\left(S_{\alpha}\right)}$. Thus

$$
\left(V_{\text {extra }}\right)_{--}-\left(V_{\text {main }}\right)_{-+} H_{0}^{-1}\left(V_{\text {main }}\right)_{+-}=\bar{H}_{\text {bos }}-\sum_{\alpha=1}^{r} p_{\alpha} \overline{D\left(S_{\alpha}\right)}=\bar{H}_{\text {target }},
$$

that is, all conditions of Lemma 5 are satisfied.

We have proved that any Hamiltonian $H_{\text {target }} \in \mathrm{HCB}_{2}(n, m, J)$ with $d$ extra diagonal terms $-p_{\alpha} D\left(S_{\alpha}\right)$ such that $0 \leq p_{\alpha} \leq J$ can be simulated with an error $(\eta, \epsilon)$ by the Hamiltonian $H_{\text {sim }} \in$ $\mathrm{HCB}_{2}\left(n^{\prime}, m^{\prime}, J^{\prime}\right)$, where $n^{\prime}=n+2 d, m^{\prime}=m+d$, and $J^{\prime}=\operatorname{poly}\left(n, J, \epsilon^{-1}, \eta^{-1}\right)$. The simulation uses an encoding $\mathcal{E}$ that maps basis vectors to basis vectors. We shall absorb the extra diagonal terms into the Hamiltonian $H_{\text {diag }}$ in all subsequent reductions. 


\section{Reduction from range-2 bosons to range- 1 bosons}

In this section we construct a range-2 HCB simulator for a range-1 HCB model. It involves a secondorder reduction. Consider a target Hamiltonian $H_{\text {target }} \in \mathrm{HCB}(n, m, J)$ describing the $m$-particle sector of range- 1 hard-core bosons on some graph $G=(U, E)$ with $n$ nodes,

$$
H_{\text {target }}=-\sum_{(u, v) \in E} t_{u, v} W_{u, v}+H_{\text {diag }} .
$$

The range-2 HCB simulator will be defined on an extended graph $G^{\prime}=\left(U^{\prime}, E^{\prime}\right)$ obtained from $G$ by placing an extra node at the center of every edge of $G$, see Fig. 6 for an example. The extra node located at the center of an edge $(u, v) \in E$ will be denoted $u+v$. Then the extended graph $G^{\prime}$ has a set of nodes

$$
U^{\prime}=U \cup U^{+}, \quad U^{+}=\{u+v:(u, v) \in E\}
$$
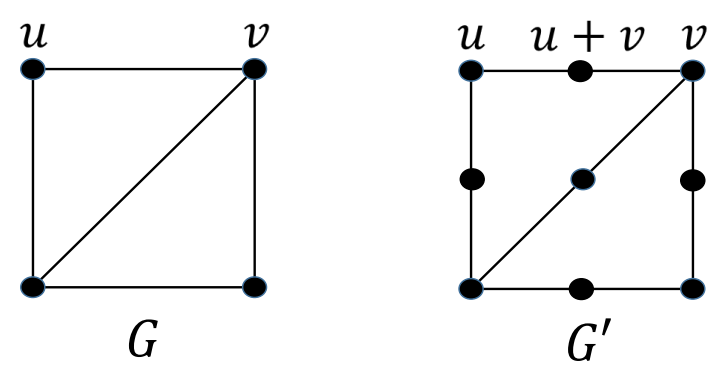

Figure 6: Construction of the extended graph $G^{\prime}=\left(U^{\prime}, E^{\prime}\right)$ for the range-2 HCB simulator starting from the graph $G=(U, E)$ for the target range-1 HCB.

The simulator and the target models have the same number of particles $m$. Thus the simulator has Hilbert space $\mathcal{B}_{m, 2}\left(G^{\prime}\right)$ spanned by 2 -sparse $m$-node subsets in the graph $G^{\prime}$. Define a simulator Hamiltonian as $H_{\text {sim }}=\Delta H_{0}+V$, where

$$
H_{0}=\sum_{w \in U^{+}} n_{w}
$$

penalizes particles that occupy nodes located at the centers of edges of $G$. We choose the perturbation as $V=\Delta^{1 / 2} V_{\text {main }}+V_{\text {extra }}$, where

$$
\begin{gathered}
V_{\text {main }}=-\sum_{(u, v) \in E^{\prime}} t_{u, v}^{1 / 2} W_{u, v}, \\
V_{\text {extra }}=H_{\text {diag }}+\sum_{(u, v) \in E} t_{u, v}\left(n_{u}-n_{v}\right)^{2} .
\end{gathered}
$$


The sum in $V_{\text {extra }}$ runs over pairs of nodes $u, v \in U$ considered as nodes of $G^{\prime}$. Clearly, $H_{0}$ has zero ground state energy and its ground subspace is spanned by subsets of nodes $S \subseteq U^{\prime}$ such that $S \cap U^{+}=\emptyset$ and $|S|=m$. Note that any distinct nodes of $S$ are automatically separated by at least two edges of $G^{\prime}$, that is, $S$ is a 2-sparse subset. Given any subset of nodes $S \subseteq U$ in the graph $G$ such that $|S|=m$, let $\mathcal{E}(S)$ be the corresponding subset of nodes in the graph $G^{\prime}$. We define the encoding $\mathcal{E}: \mathcal{B}_{m}(G) \rightarrow \mathcal{B}_{m, 2}\left(G^{\prime}\right)$ such that $\mathcal{E}|S\rangle=|\mathcal{E}(S)\rangle$. The above shows that $\operatorname{Im}(\mathcal{E})$ coincides with the ground subspace of $H_{0}$.

Let us check that the perturbation $V$ satisfies the conditions of Lemma 5. First, we note that $\left(V_{\text {main }}\right)_{--}=0$. Indeed, suppose $S \subseteq U^{\prime}$ is a ground state of $H_{0}$. Then $S \cap U^{+}=\emptyset$. Thus $V_{\text {main }}$ can only move a particle from some node $u \in U$ to some node $v \in U^{+}$which produces an excited state of $H_{0}$. The operator $V_{\text {extra }}$ is block diagonal because it is diagonal.

Let us now check condition Eq. (32) of Lemma 5. Consider any ground state of $H_{0}$, that is, $m$-node subset $S \subseteq U^{\prime}$ such that $S \cap U^{+}=\emptyset$. We claim that

$$
\left(V_{\text {main }}\right)_{+-}|S\rangle=-\sum_{u \in S} \sum_{(u, v) \in E} t_{u, v}^{1 / 2}\left(1-n_{v}\right) W_{u, u+v}|S\rangle
$$

Indeed, the hopping terms in $V_{\text {main }}$ can only move a particle from some node $u \in S$ to some node $u+v$ such that $(u, v) \in E$ and such that the resulting configuration of particles is 2-sparse. The latter condition is satisfied iff $n_{v}=0$. Taking into account that $W_{u, u+v}|S\rangle$ is an eigenvector of $H_{0}^{-1}$ with an eigenvalue one, we get

$$
\left(V_{\text {main }}\right)_{-+} H_{0}^{-1}\left(V_{\text {main }}\right)_{+-}=\sum_{(u, v) \in E} t_{u, v} W_{u, v}+\sum_{u \in U} \sum_{(u, v) \in E} t_{u, v} n_{u}\left(1-n_{v}\right) .
$$

Here the last term accounts for double-hopping processes where $\left(V_{\text {main }}\right)_{+-}$moves a particle from $u$ to $u+v$ and $\left(V_{\text {main }}\right)_{-+}$returns the particle back to $u$. Using the identity $n_{u}\left(1-n_{v}\right)+n_{v}\left(1-n_{u}\right)=$ $\left(n_{u}-n_{v}\right)^{2}$ one gets

$$
\left(V_{\text {main }}\right)_{-+} H_{0}^{-1}\left(V_{\text {main }}\right)_{+-}=\sum_{(u, v) \in E} t_{u, v} W_{u, v}+\sum_{(u, v) \in E} t_{u, v}\left(n_{u}-n_{v}\right)^{2}
$$

The last term is exactly cancelled by $V_{\text {extra }}$ which proves condition Eq. (32) of Lemma 5 . Note that in this case the logical operators $\bar{W}_{u, v}$ and $\bar{n}_{u}$ coincide with $W_{u, v}$ and $n_{u}$ since we encode each particle of the target model by a single particle in the simulator model.

To conclude, we have proved that any Hamiltonian $H_{\text {target }} \in \mathrm{HCB}(n, m, J)$ can be simulated with an error $(\eta, \epsilon)$ by the Hamiltonian $H_{\text {sim }} \in \mathrm{HCB}_{2}\left(n^{\prime}, m, J^{\prime}\right)$, where $n^{\prime}=O\left(n^{2}\right)$ and $J^{\prime}=$ poly $\left(n, J, \epsilon^{-1}, \eta^{-1}\right)$. The simulation uses an encoding $\mathcal{E}$ that maps basis vectors to basis vectors. 


\section{Range- 1 bosons with a controlled hopping}

Consider a graph $G=(U, E)$ with $n$ nodes and a Hamiltonian $H_{\text {bos }} \in \operatorname{HCB}(n, m, J)$ describing the $m$-particle sector of the range-1 HCB model on the graph $G$. Suppose our target Hamiltonian is

$$
H_{\text {target }}=H_{b o s}-\sum_{(c ; u, v)} t_{c ; u, v} n_{c} W_{u, v}
$$

where the sum runs over all triples of nodes $(c ; u, v)$ such that $c \in U,(u, v) \in E$, and $c \notin\{u, v\}$. The term $n_{c} W_{u, v}$ describes a controlled hopping process where the presence of particle at the node $c$ controls whether the hopping between nodes $u, v$ is turned on or off. The coefficients $t_{c ; u, v}$ are the controlled hopping amplitudes. We shall always assume that $t_{c ; u, v} \geq 0$. The Hamiltonian $H_{\text {target }}$ acts on the $m$-particle sector $\mathcal{B}_{m}(G)$. Let $\operatorname{HCB}^{*}(n, m, J)$ be the set of Hamiltonians $H_{\text {target }}$ defined above where $0 \leq t_{c ; u, v} \leq J$. In this section we show how to simulate $H_{\text {target }}$ by the standard range- 1 HCB. The simulation involves a composition of a first-order and a second-order reduction.

The simulator model will be defined on a graph $G^{\prime}=\left(U^{\prime}, E^{\prime}\right)$ obtained from $G$ by adding certain extra nodes and extra edges. Namely, for each triple $(c ; u, v)$ that appears in Eq. (85) we add an extra node $a(c ; u, v)$ and a pair of extra edges connecting $a(c ; u, v)$ to $u$ and $v$. Let $n^{\prime}=\left|U^{\prime}\right|$ be the number of nodes in the extended graph and $U^{+} \subseteq U^{\prime}$ be the set of all extra nodes $a(c ; u, v)$. The simulator and the target models have the same number of particles $m$.

Our first reduction has a simulator Hamiltonian

$$
\tilde{H}_{\text {sim }}=\tilde{\Delta} \tilde{H}_{0}+\tilde{V}, \quad \tilde{H}_{0}=\sum_{(c ; u, v)}\left(I-n_{c}\right) n_{a(c ; u, v)}
$$

where $\tilde{V} \in \operatorname{HCB}\left(n^{\prime}, m, \tilde{J}\right)$ will be chosen at the next reduction. The Hamiltonian $\tilde{H}_{\text {sim }}$ acts on the Hilbert space $\mathcal{B}_{m}\left(\mathcal{G}^{\prime}\right)$. Let $\tilde{\mathcal{H}}_{-}$be the ground subspace of $\tilde{H}_{0}$. Obviously, $\tilde{\mathcal{H}}_{-}$is spanned by configurations of particles such that a node $a(c ; u, v)$ can be occupied only if $c$ is occupied. This property must hold for each extra node $a(c ; u, v)$. Lemma 4 shows that $\tilde{H}_{\text {sim }}$ can simulate the

restriction of any Hamiltonian from $\operatorname{HCB}\left(n^{\prime}, m, \tilde{J}\right)$ onto the subspace $\tilde{\mathcal{H}}_{-}$. In the rest of this section we assume that our full Hilbert space is $\mathcal{H}=\tilde{\mathcal{H}}_{-}$. The above simulation uses the trivial encoding, that is, $\mathcal{E}|S\rangle=|S\rangle$ if $S \subseteq U^{\prime}$ is a ground state of $\tilde{H}_{0}$ and $\mathcal{E}|S\rangle=0$ otherwise.

Our second reduction has a simulator Hamiltonian

$$
H_{\text {sim }}=\Delta H_{0}+V, \quad H_{0}=\Delta \sum_{a \in U^{+}} n_{a}, \quad V=\Delta^{1 / 2} V_{\text {main }}+V_{\text {extra }}
$$

where

$$
\begin{gathered}
V_{\text {main }}=-\sum_{(c ; u, v)}\left(t_{c ; u, v}\right)^{1 / 2}\left(W_{u, a(c ; u, v)}+W_{v, a(c ; u, v)}\right), \\
V_{\text {extra }}=H_{b o s}+\sum_{(c ; u, v)} t_{c ; u, v} n_{c}\left(n_{u}+n_{v}\right) .
\end{gathered}
$$


Here all operators are restricted to the subspace $\mathcal{H}$ defined above. We choose an encoding $\mathcal{E}$ : $\mathcal{B}_{m}(G) \rightarrow \mathcal{H}$ that maps subsets of nodes in the graph $G$ to the corresponding subsets of nodes in the extended graph $G^{\prime}$. Obviously, $S \subseteq U^{\prime}$ is a ground state of $H_{0}$ iff $|S|=m$ and $S \cap U^{+}=\emptyset$, that is, all the extra nodes $a(c ; u, v)$ are empty. Thus $\operatorname{Im}(\mathcal{E})$ coincides with the ground subspace of $H_{0}$.

Let us check that the perturbation $V$ satisfies all conditions of Lemma 5 . We note that $\left(V_{\text {main }}\right)_{--}=$ 0 since $V_{\text {main }}$ can only move a particle from (to) some ancillary node $a(c ; u, v)$ which must be empty in any ground state of $H_{0}$. The Hamiltonian $V_{\text {extra }}$ is block-diagonal since $H_{b o s}$ acts trivially on all ancillary nodes whereas the second term in $V_{\text {extra }}$ is diagonal. It remains to check condition Eq. (32) of Lemma 5. Let $S \subseteq U^{\prime}$ be any ground state of $H_{0}$. Then $S \cap U^{+}=\emptyset$. We claim that $W_{u, a(c ; u, v)}|S\rangle=0$ unless $n_{c}=1$. Indeed, if $n_{c}=0$ and $n_{u}=0$ then both nodes $u$ and $a(c ; u, v)$ are empty. If $n_{c}=0$ and $n_{u}=1$ then $W_{u, a(c ; u, v)}$ moves a particle from $u$ to $a(c ; u, v)$. However, a state in which the node $a(c ; u, v)$ is occupied and the node $c$ is empty is orthogonal to the subspace $\mathcal{H}$. Since the simulator model is restricted to $\mathcal{H}$, we have $W_{u, a(c ; u, v)}|S\rangle=0$ in both cases. In the remaining case, $n_{c}=1$, one has $W_{u, a(c ; u, v)}|S\rangle=\left|S^{\prime}\right\rangle$, where $S^{\prime}=(S \backslash u) \cup a(c ; u, v)$. The above shows that

$$
\left(V_{\text {main }}\right)_{-+} H_{0}^{-1}\left(V_{\text {main }}\right)_{+-}=\sum_{(c ; u, v)} t_{c ; u, v} n_{c}\left(W_{u, v}+n_{u}+n_{v}\right) .
$$

Here the first term describes processes where $\left(V_{\text {main }}\right)_{+-}$moves a particle from $u$ to $a(c ; u, v)$ and $\left(V_{\text {main }}\right)_{-+}$moves the particle from $a(c ; u, v)$ to $v$. The last two terms describe processes where $\left(V_{\text {main }}\right)_{+-}$moves a particle from $u$ to $a(c ; u, v)$ and $\left(V_{\text {main }}\right)_{-+}$returns the particle back to $u$. The last two terms in Eq. (90) are canceled by $V_{\text {extra }}$. This proves condition Eq. (32) of Lemma 5 . Note that in this case the logical operators $\bar{W}_{u, v}$ and $\bar{n}_{u}$ coincide with $W_{u, v}$ and $n_{u}$ since we encode each particle of the target model by a single particle in the simulator model.

Combining Lemmas $3,4,5$ we conclude that any Hamiltonian $H_{\text {target }} \in \operatorname{HCB}^{*}(n, m, J)$ can be

simulated with an error $(\eta, \epsilon)$ by the Hamiltonian $\tilde{H}_{\text {sim }} \in \operatorname{HCB}\left(n^{\prime}, m, J^{\prime}\right)$ where $n^{\prime}=O\left(n^{3}\right)$ and $J^{\prime}=\operatorname{poly}\left(n, J, \epsilon^{-1}, \eta^{-1}\right)$. The simulation uses an encoding $\mathcal{E}$ that maps basis vectors to basis vectors.

\section{From range-1 bosons to 2-local stoquastic Hamiltonians}

Let us start from a simple classification of two-qubit stoquastic interactions. In this section we use the standard $|0\rangle,|1\rangle$ basis for a single qubit and the corresponding product basis for $n$ qubits.

Lemma 9. Let $H$ be a two-qubit hermitian operator such that $H$ has real matrix elements in the standard basis and all off-diagonal matrix elements of $H$ are non-positive. Then $H$ can be written as a sum of some diagonal two-qubit Hamiltonian $H_{\text {diag }}$ and a convex linear combination of operators

$$
\begin{aligned}
& \text { 1. }-X \otimes|0\rangle\langle 0| \text { and }-X \otimes|1\rangle\langle 1| \\
& \text { 2. }-|0\rangle\langle 0| \otimes X \text { and }-|1\rangle\langle 1| \otimes X \\
& \text { 3. }-X \otimes X-Y \otimes Y
\end{aligned}
$$




$$
\text { 4. }-X \otimes X+Y \otimes Y
$$

Proof. Let $G \equiv-H$. Since $G$ has real matrix elements, the expansion of $G$ in the basis of Pauli operators contains only the terms with even number of $Y$ 's. Thus

$$
G=-H_{\text {diag }}+h_{X I} X \otimes I+h_{I X} I \otimes X+h_{X X} X \otimes X+h_{X Z} X \otimes Z+h_{Z X} Z \otimes X+h_{Y Y} Y \otimes Y,
$$

where $H_{\text {diag }}$ is some diagonal Hamiltonian. From

$$
\langle 0,0|G| 1,1\rangle=h_{X X}-h_{Y Y} \geq 0 \quad \text { and } \quad\langle 0,1|G| 1,0\rangle=h_{X X}+h_{Y Y} \geq 0
$$

one gets

$$
h_{X X} X \otimes X+h_{Y Y} Y \otimes Y=p(X \otimes X+Y \otimes Y)+q(X \otimes X-Y \otimes Y),
$$

where $p=\left(h_{X X}+h_{Y Y}\right) / 2$ and $q=\left(h_{X X}-h_{Y Y}\right) / 2$ are non-negative coefficients. From

$$
\langle 0,0|G| 1,0\rangle=h_{X I}+h_{X Z} \geq 0 \quad \text { and } \quad\langle 0,1|G| 1,1\rangle=h_{X I}-h_{X Z} \geq 0
$$

one gets

$$
h_{X I} X \otimes I+h_{X Z} X \otimes Z=p X \otimes|0\rangle\langle 0|+q X \otimes| 1\rangle\langle 1|,
$$

where $p=h_{X I}+h_{X Z}$ and $q=h_{X I}-h_{X Z}$ are non-negative coefficients. Similar calculation shows that

$$
h_{I X} I \otimes X+h_{Z X} Z \otimes X=p|0\rangle\langle 0|\otimes X+q| 1\rangle\langle 1| \otimes X,
$$

where $p=h_{I X}+h_{Z X}$ and $q=h_{I X}-h_{Z X}$ are non-negative coefficients. The lemma now follows from Eqs. (91-94).

Let $H_{\text {target }} \in \operatorname{StoqLH}(n, J)$ be some fixed 2-local stoquastic Hamiltonian on $n$ qubits. Our goal is to simulate $H_{\text {target }}$ by some Hamiltonian $H_{\text {sim }} \in \mathrm{HCB}^{*}\left(n^{\prime}, m, J^{\prime}\right)$. Recall that the latter describes the $m$-particle sector of range- 1 hard-core bosons with a controlled hopping on some graph $G=(U, E)$ with $n^{\prime}$ nodes, see Section 10. We shall represent the $j$-th qubit of the target model by a pair of nodes $\{2 j-1,2 j\} \subseteq U$. The two basis states $|0\rangle$ and $|1\rangle$ of the $j$-th qubit are represented by a particle located at the node $2 j-1$ and $2 j$ respectively (the dual rail representation). Thus the number of particles in the simulator model is $m=n$.

For the sake of clarity we shall first explain how to construct an $\mathrm{HCB}^{*}$ simulator individually for each two-qubit stoquastic interaction listed in Lemma 9. Thus we shall first consider the case $n=m=2$. We shall simulate interactions (1) and (2) using a first-order reduction. Interactions (3) and (4) will require a composition of a first-order and a third-order reductions. Then we shall explain how to combine the simulators together. To avoid interference between simulators we shall introduce some ancillary nodes such that a simulator is activated only if the corresponding ancillary node is occupied by a particle. 
Consider first the case $H_{\text {target }}=-p X \otimes|0\rangle\langle 0|$ with $p>0$. The HCB* simulator is defined on a graph $G=(U, E)$, where $U=\{1,2,3,4\}$ and $E=\{1,2\}$. The total number of particles is $m=2$, so that the simulator Hilbert space is $\mathcal{B}_{2}(G)$. The simulator Hamiltonian is chosen as

$$
\begin{gathered}
H_{\text {sim }}=\Delta H_{0}+V, \quad H_{0}=n_{1} n_{2}+n_{3} n_{4}, \\
V=-p n_{3} W_{1,2} .
\end{gathered}
$$

Ground states of $H_{0}$ are subsets of nodes $\{i, j\} \subseteq U$, where $i \in\{1,2\}$ and $j \in\{3,4\}$. The ground subspace of $H_{0}$ encodes two logical qubits as follows:

$$
|\overline{0,0}\rangle=|1,3\rangle, \quad|\overline{0,1}\rangle=|1,4\rangle, \quad|\overline{1,0}\rangle=|2,3\rangle, \quad|\overline{1,1}\rangle=|2,4\rangle .
$$

This is analogous to applying the dual-rail representation to each qubit. Obviously, $V$ commutes with $H_{0}$. Note that $W_{1,2}$ implements the logical $\bar{X}$ on the first qubit and $n_{3}$ implements the logical operator $|\overline{0}\rangle\langle\overline{0}|$ on the second qubit. Lemma 4 implies that $H_{\text {sim }}$ can simulate the restriction of $V$ onto the logical subspace, that is, $V_{--}=-p \bar{X} \otimes|\overline{0}\rangle\langle\overline{0}|$. This is the desired target Hamiltonian. Using the same method one can simulate all elementary interactions (1) and (2) in Lemma 9.

Next consider the case

$$
H_{\text {target }}=-(p / 2)(X \otimes X+Y \otimes Y)=-p(|1,0\rangle\langle 0,1|+| 0,1\rangle\langle 1,0|), \quad p>0 .
$$

The $\mathrm{HCB}^{*}$ simulator will be defined on a graph $G=(U, E)$ where $U=\{1,2,3,4, a\}$, see Fig. 7 . We choose the total number of particles $m=2$. The simulation involves a composition of a first-order and a second-order reductions.

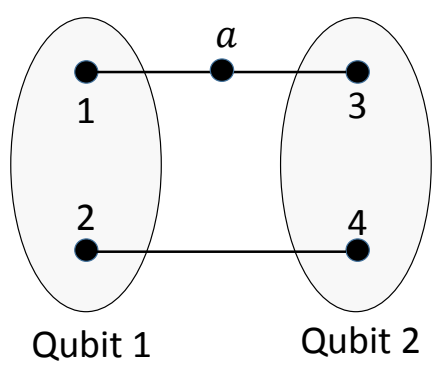

Figure 7: Graph $G=(U, E)$ of the $\mathrm{HCB}^{*}$ simulator for the target Hamiltonian $-X \otimes X-Y \otimes Y$. The total number of particles is $m=2$.

Our first reduction has a simulator Hamiltonian

$$
\tilde{H}_{\text {sim }}=\tilde{\Delta} \tilde{H}_{0}+\tilde{V}, \quad \tilde{H}_{0}=n_{1} n_{2}+n_{3} n_{4}, \quad \tilde{V} \in \operatorname{HCB}^{*}(5,2, \tilde{J}) .
$$

All above operators act on the Hilbert space $\mathcal{B}_{2}(G)$. The perturbation $\tilde{V}$ will be chosen at the next reduction. Let $\mathcal{H}$ be the ground subspace of $\tilde{H}_{0}$. It is spanned by configurations of particles such 
that each qubit $\{1,2\}$ and $\{3,4\}$ contains at most one particle. Lemma 4 implies that $\tilde{H}_{\text {sim }}$ can simulate the restriction of any $\mathrm{HCB}^{*}$ Hamiltonian $\tilde{V}$ onto the subspace $\mathcal{H}$. Below we assume that our full Hilbert space is $\mathcal{H}$.

Our second reduction has a simulator Hamiltonian

$$
H_{\text {sim }}=\Delta H_{0}+V, \quad H_{0}=n_{a}, \quad V=\Delta^{2 / 3} V_{\text {main }}+\Delta^{1 / 3} \tilde{V}_{\text {extra }}
$$

where

$$
\begin{gathered}
V_{\text {main }}=-p^{1 / 3}\left(W_{1, a}+W_{3, a}+n_{a} W_{2,4}\right), \\
\tilde{V}_{\text {extra }}=p^{2 / 3}\left(2 n_{1} n_{3}+n_{1} n_{4}+n_{2} n_{3}\right) .
\end{gathered}
$$

Here all operators are restricted to the subspace $\mathcal{H}$ with at most one particle per qubit. Note that $H_{\text {sim }}$ is an HCB* Hamiltonian. The ground subspace of $H_{0}$ encodes two logical qubits according to Eq. (97). Let us check that the perturbation satisfies all conditions of Lemma 6 . Note that $\left(V_{\text {main }}\right)_{--}=0$ since $V_{\text {main }}$ can only move a particle from (to) the ancillary node $a$. The last term in $V_{\text {main }}$ does not contribute to $\left(V_{\text {main }}\right)_{--}$since $n_{a}=0$ for any ground state of $H_{0}$. Obviously, $\tilde{V}_{\text {extra }}$ is block-diagonal.

Let us check condition Eq. (33) of Lemma 6. Informally, it says that the third-order hopping process generated by $V_{\text {main }}$ must implement the logical hopping operator between the two logical qubits. For example, suppose the initial state is $|\overline{0,1}\rangle=|1,4\rangle$. Then $\left(V_{\text {main }}\right)_{+-}$moves a particle from 1 to $a$ by applying $W_{1, a}$, then $\left(V_{\text {main }}\right)_{++}$moves a particle from 4 to 2 by applying $n_{a} W_{2,4}$, and then $\left(V_{\text {main }}\right)_{-+}$moves a particle from $a$ to 3 by applying $W_{3, a}$. This produces the correct final state $|\overline{1,0}\rangle=|2,3\rangle$. More formally, one can easily check that

$$
\begin{aligned}
& \left(V_{\text {main }}\right)_{+-}|\overline{1,1}\rangle=\left(V_{\text {main }}\right)_{+-}|2,4\rangle=0 \\
& \left(V_{\text {main }}\right)_{+-}|\overline{0,0}\rangle=\left(V_{\text {main }}\right)_{+-}|1,3\rangle=-p^{1 / 3}(|3, a\rangle+|1, a\rangle), \\
& \left(V_{\text {main }}\right)_{+-}|\overline{0,1}\rangle=\left(V_{\text {main }}\right)_{+-}|1,4\rangle=-p^{1 / 3}|4, a\rangle \\
& \left(V_{\text {main }}\right)_{+-}|\overline{1,0}\rangle=\left(V_{\text {main }}\right)_{+-}|2,3\rangle=-p^{1 / 3}|2, a\rangle .
\end{aligned}
$$

From this one easily gets

$$
\left(V_{\text {main }}\right)_{-+} H_{0}^{-1}\left(V_{\text {main }}\right)_{++} H_{0}^{-1}\left(V_{\text {main }}\right)_{+-}=-p|\overline{0,1}\rangle\langle\overline{1,0}|-p| \overline{1,0}\rangle\langle\overline{0,1}|=\bar{H}_{\text {target }} .
$$

This proves condition Eq. (33). A similar calculation shows that

$$
\left(V_{\text {main }}\right)_{-+} H_{0}^{-1}\left(V_{\text {main }}\right)_{+-}=p^{2 / 3}(2|\overline{0,0}\rangle\langle\overline{0,0}|+| \overline{0,1}\rangle\langle\overline{0,1}|+| \overline{1,0}\rangle\langle\overline{1,0}|)=p^{2 / 3}\left(2 n_{1} n_{3}+n_{1} n_{4}+n_{2} n_{3}\right)
$$

which proves condition Eq. (34).

To compose the two reductions we extend $H_{\text {sim }}$ defined in Eq. (100) to the full Hilbert space $\mathcal{B}_{2}(G)$ and substitute $\tilde{V}=H_{\text {sim }}$ into Eq. (99). Combining Lemmas 3,4,6 we conclude that $H_{\text {target }}$ can be simulated with an error $(\eta, \epsilon)$ by $\tilde{H}_{\text {sim }} \in \operatorname{HCB}^{*}\left(5,2, J^{\prime}\right)$ where $J^{\prime}=\operatorname{poly}\left(p, \eta^{-1}, \epsilon^{-1}\right)$. The simulation uses the dual rail encoding $\mathcal{E}$ defined in Eq. (97). 
Finally, consider the case $H_{\text {target }}=-p X \otimes X+p Y \otimes Y$, where $p>0$. This Hamiltonian can be obtained from $H_{\text {target }}=-p(X \otimes X+Y \otimes Y)$ by conjugating the second qubit with $X$. This is equivalent to exchanging nodes 3 and 4 in the reduction described above. Hence we have constructed an $\mathrm{HCB}^{*}$ simulator for all elementary stoquastic interactions.

Suppose now that $H_{\text {target }} \in \operatorname{StoqLH}(n, J)$ is a general 2-local stoquastic Hamiltonian on $n$ qubits. By Lemma 9,

$$
H_{\text {target }}=H_{\text {diag }}+\sum_{\alpha=1}^{m} p_{\alpha} H_{\alpha},
$$

where $H_{\text {diag }}$ is a diagonal 2-local Hamltonian with terms proportional to $n_{u}$ and $n_{u} n_{v}$, where $p_{\alpha}>0$ are some coefficients, and each term $H_{\alpha}$ is one of the four elementary stoquastic interactions applied to some pair of qubits. Obviously, the number of terms is $m \leq$ poly $(n)$. The corresponding HCB* simulator $H_{\text {sim }}$ will be defined on a graph $G=(U, E)$ with $2 n+m^{\prime}$ nodes, where $m^{\prime} \leq m$ is the number of interactions of type (3) or (4) in $H_{\text {target }}$, see Lemma 9. Without loss of generality, the first $m^{\prime}$ interactions $H_{\alpha}$ are of type (3) or (4). The total number of particles in the HCB* simulator will be $m=n$, so that the Hilbert space of the simulator is $\mathcal{B}_{n}(G)$. We shall need a composition of a first-order and a third-order reductions.

Let us first define the set of nodes of $G$. Each qubit $i$ gives rise to a pair of nodes $t(i)$ and $b(i)$ which form the dual-rail representation of the qubit. We encode the basis states $|0\rangle$ and $|1\rangle$ by a single particle located at the node $t(i)$ and $b(i)$ respectively. Each interaction $H_{\alpha}$ of type (3) or (4) gives rise to an extra node $a(\alpha)$ in $G$. This is the ancillary node used in the graph shown on Fig. 7.

The set of edges of $G$ is defined as follows. For each interaction $H_{\alpha}$ of type (3) coupling qubits $i, j$ we add an edge connecting nodes $t(i)$ and $a(\alpha)$, an edge connecting nodes $a(\alpha)$ and $t(j)$, and an edge connecting nodes $b(i)$ and $b(j)$. The last edge represents a controlled hopping with a control node $a(\alpha)$. For each interaction $H_{\beta}$ of type (4) coupling qubits $i, j$ we add an edge connecting nodes $t(i)$ and $a(\beta)$, an edge connecting nodes $a(\beta)$ and $b(j)$, and an edge connecting nodes $b(i)$ and $t(j)$. The last edge represents a controlled hopping with a control node $a(\beta)$. The resulting subgraph of $G$ is shown on Fig. 8. This completes definition of the graph $G$ for the HCB simulator.

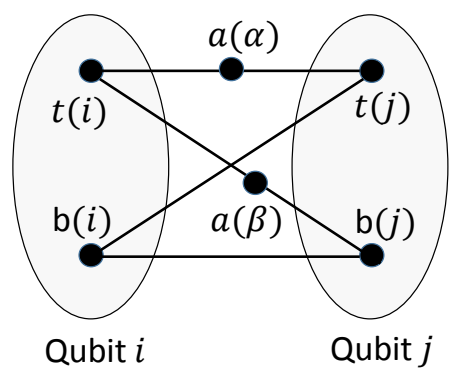

Figure 8: Construction of the graph $G$ for the HCB simulator. Each qubit $i$ is represented by a pair of nodes $t(i), b(i)$ using the dual-rail representation. The ancillary node $a(\alpha)$ controls hopping between $b(i)$ and $b(j)$. The ancillary node $a(\beta)$ controls hopping between $b(i)$ and $t(j)$. 
Our first reduction has a simulator Hamiltonian

$$
\tilde{H}_{\text {sim }}=\tilde{\Delta} \tilde{H}_{0}+\tilde{V}, \quad \tilde{H}_{0}=\sum_{i=1}^{n} n_{t(i)} n_{b(i)}, \quad \tilde{V} \in \operatorname{HCB}^{*}\left(2 n+m^{\prime}, n, \tilde{J}\right) .
$$

All above operators act on the Hilbert space $\mathcal{B}_{n}(G)$. The perturbation $\tilde{V}$ will be chosen at the next reduction. Let $\mathcal{H}$ be the ground subspace of $\tilde{H}_{0}$. It is is spanned by configurations of particles such that each qubit $\{t(i), b(i)\}$ contains at most one particle. Lemma 4 implies that $\tilde{H}_{\text {sim }}$ can simulate the restriction of any $\mathrm{HCB}^{*}$ Hamiltonian $\tilde{V}$ onto the subspace $\mathcal{H}$. Below we assume that our full Hilbert space is $\mathcal{H}$.

Our second reduction has a simulator Hamiltonian

$$
H_{\text {sim }}=\Delta H_{0}+V, \quad H_{0}=\sum_{\alpha=1}^{m^{\prime}} n_{a(\alpha)}, \quad V=\Delta^{2 / 3} V_{\text {main }}+\Delta^{1 / 3} \tilde{V}_{\text {extra }}+V_{\text {extra }}
$$

Since the total number of particles is $n$ and each qubit may contain at most one particle, the ground subspace of $H_{0}$ is spanned by states with exactly one particle per qubit. It encodes $n$ logical qubits under the dual rail representation. The perturbation operators are defined as the sums of respective perturbation operators over all individual simulators. More formally,

$$
V_{\text {main }}=\sum_{\alpha=1}^{m^{\prime}} V_{\text {main }}^{\alpha}, \quad \tilde{V}_{\text {extra }}=\sum_{\alpha=1}^{m^{\prime}} \tilde{V}_{\text {extra }}^{\alpha}, \quad V_{\text {extra }}=H_{\text {diag }}+\sum_{\alpha=m^{\prime}+1}^{m} V^{\alpha},
$$

where the perturbation operators carrying an index $\alpha$ are defined by Eqs. $(96,101,102)$, depending on the type of the interaction $H_{\alpha}$, with $p$ replaced by $p_{\alpha}$, with the nodes $\{1,2\}$ replaced by the nodes of the first logical qubit acted upon by $H_{\alpha}$, and with the nodes $\{3,4\}$ replaced by the nodes of the second logical qubit acted upon by $H_{\alpha}$.

Let us check that the perturbation $V$ satisfies all conditions of Lemma 6 . By definition, $V_{\text {extra }}$ and $\tilde{V}_{\text {extra }}$ act trivially on the ancillary nodes $a(\alpha)$ and thus they are block diagonal. Any term in $V_{\text {main }}$ moves a particle to (from) some ancillary node $a(\alpha)$. Since all these nodes must be empty in the ground subspace of $H_{0}$, one has $\left(V_{\text {main }}\right)_{--}=0$.

Let us now check condition Eq. (34). We claim that

$$
\left(V_{\text {main }}\right)_{-+} H_{0}^{-1}\left(V_{\text {main }}\right)_{+-}=\sum_{\alpha=1}^{m^{\prime}}\left(V_{\text {main }}^{\alpha}\right)_{-+} H_{0}^{-1}\left(V_{\text {main }}^{\alpha}\right)_{+-}
$$

Indeed, any term in $\left(V_{\text {main }}\right)_{+-}$must move a particle from some qubit node $u \in\{t(i), b(i)\}$ to some ancillary node $a(\alpha)$. In order to return the system to the ground subspace of $H_{0}$, the factor $\left(V_{\text {main }}\right)_{-+}$ must move the particle from $a(\alpha)$ to $u$. Thus $\left(V_{\text {main }}^{\alpha}\right)_{-+} H_{0}^{-1}\left(V_{\text {main }}^{\beta}\right)_{+-}=0$ for $\alpha \neq \beta$. This implies Eq. (109). Since $V_{\text {main }}^{\alpha}$ and $\tilde{V}_{\text {extra }}^{\alpha}$ satisfy condition Eq. (34) for each individual simulator, Eq. (109) implies that $V_{\text {main }}$ and $\tilde{V}_{\text {extra }}$ also satisfy condition Eq. (34). 
Next let us check condition Eq. (33) of Lemma 6. We claim that

$$
\left(V_{\text {main }}\right)_{-+} H_{0}^{-1}\left(V_{\text {main }}\right)_{++} H_{0}^{-1}\left(V_{\text {main }}\right)_{+-}=\sum_{\alpha=1}^{m}\left(V_{\text {main }}^{\alpha}\right)_{-+} H_{0}^{-1}\left(V_{\text {main }}^{\alpha}\right)_{++} H_{0}^{-1}\left(V_{\text {main }}^{\alpha}\right)_{+-}
$$

Indeed, suppose $\left(V_{\text {main }}\right)_{+-}$moves a particle from a qubit node $u \in\{t(i), b(i)\}$ to some ancillary node $a(\alpha)$. For concreteness, assume that $H_{\alpha}$ is an interaction of type (3) coupling qubits $i, j$ and $u=t(i)$. The factor $\left(V_{\text {main }}\right)_{++}$can either apply the controlled hopping term proportional to $n_{a(\alpha)} W_{b(i), b(j)}$ or move a particle from some other qubit node $v \in\left\{t\left(i^{\prime}\right), b\left(i^{\prime}\right)\right\}$ to some ancillary node $a(\beta)$ with $\alpha \neq \beta$. In the latter case, however, we create two excited ancillary nodes so that $\left(V_{\text {main }}\right)_{-+}$will not be able to return the system back to the ground subspace of $H_{0}$. In the former case $\left(V_{\text {main }}\right)_{-+}$can return the system to the ground subspace of $H_{0}$ only by moving the particle from $a(\alpha)$ to some node of qubit $i$ or $j$. This proves Eq. (110). Since $V_{\text {main }}^{\alpha}$ satisfies condition Eq. (33) for each individual simulator with $V_{\text {extra }}^{\alpha}=0$, Eq. (110) implies that

$$
\left(V_{\text {main }}\right)_{-+} H_{0}^{-1}\left(V_{\text {main }}\right)_{++} H_{0}^{-1}\left(V_{\text {main }}\right)_{+-}=\sum_{\alpha=1}^{m^{\prime}} p_{\alpha} \bar{H}_{\alpha} .
$$

Furthermore, one has $\left(V^{\alpha}\right)_{--}=p_{\alpha} \bar{H}_{\alpha}$ for interactions of type (1) and (2), that is, for $m^{\prime}<\alpha \leq m$. Therefore

$$
\left(V_{\text {extra }}\right)_{--}=\bar{H}_{\text {diag }}+\sum_{\alpha=m^{\prime}+1}^{m} p_{\alpha} \bar{H}_{\alpha}
$$

Combining this and Eq. (111) one arrives at

$$
\left(V_{\text {extra }}\right)_{--}+\left(V_{\text {main }}\right)_{-+} H_{0}^{-1}\left(V_{\text {main }}\right)_{++} H_{0}^{-1}\left(V_{\text {main }}\right)_{+-}=\bar{H}_{\text {target }} .
$$

Thus all conditions of Lemma 6 are satisfied.

To compose the two reductions we extend $H_{\text {sim }}$ defined in Eq. (107) to the full Hilbert space $\mathcal{B}_{n}(G)$ and substitute $\tilde{V}=H_{\text {sim }}$ into Eq. (106). Combining Lemmas 3,4,6 we conclude that any Hamiltonian $H_{\text {target }} \in \operatorname{StoqLH}(n, J)$ can be simulated with an error $(\eta, \epsilon)$ by the Hamiltonian $\tilde{H}_{\text {sim }} \in$ $\operatorname{HCB}^{*}\left(n^{\prime}, n, J^{\prime}\right)$ where $n^{\prime}=2 n+m^{\prime}=O\left(n^{2}\right)$ and $J^{\prime}=\operatorname{poly}\left(n, J, \epsilon^{-1}, \eta^{-1}\right)$. The simulation uses the dual rail encoding $\mathcal{E}$ that maps basis vectors to basis vectors.

Finally, let us extend the above reduction to $(2, k)$-local stoquastic Hamiltonians. We have to modify the simulator model by adding multi-particle interactions as described in Section 8. More precisely, consider the target Hamiltonian defined in Eq. (105) and suppose the term $H_{\text {diag }}$ contains $k$-qubit diagonal interactions with strength at most $J$. Such Hamiltonian can be written as

$$
H_{\text {diag }}=-\sum_{x \in\{0,1\}^{k}} \sum_{M \subseteq[n]} p(x, M)|x\rangle\left\langle\left. x\right|_{M},\right.
$$

where $p(x, M)$ are some real coefficients such that $|p(x, M)| \leq \operatorname{poly}(J, n)$, and $|x\rangle\left\langle\left. x\right|_{M}\right.$ is the $k$-qubit projector $|x\rangle\langle x|$ acting on a subset of qubits $M$. Performing an overall energy shift one can achieve 
$p(x, M) \geq 0$ for all $x$ and $M$. Consider any fixed projector $|x\rangle\left\langle\left. x\right|_{M}\right.$. Recall that the basis states $\left.\mid 0\right\rangle$ and $|1\rangle$ of the $i$-th qubit of the target model are encoded by a particle located at the node $t(i)$ and $b(i)$ respectively. It follows that the encoded version of a projector $|0\rangle\left\langle\left. 0\right|_{i}\right.$ can be written as $I-n_{b(i)}$. Likewise, the encoded version of a projector $|1\rangle\left\langle\left. 1\right|_{i}\right.$ can be written as $I-n_{t(i)}$. Thus the encoded version of the projector $|x\rangle\left\langle\left. x\right|_{M}\right.$ is

$$
\overline{|x\rangle\langle x|}_{M}=\prod_{i \in M: x(i)=0}\left(I-n_{b(i)}\right) \prod_{i \in M: x(i)=1}\left(I-n_{t(i)}\right) .
$$

Here $x(i)$ denotes the bit of the string $x$ associated with the $i$-th qubit. Let $S(x, M) \subseteq U$ be the union of all nodes $b(i)$ with $i \in M$ and $x(i)=0$ and all nodes $t(i)$ with $i \in M$ and $x(i)=1$. We conclude that the encoded version of $H_{\text {diag }}$ is

$$
\bar{H}_{\text {diag }}=-\sum_{x \in\{0,1\}^{k}} \sum_{M \subseteq[n]} p(x, M) \prod_{u \in S(x, M)}\left(I-n_{u}\right) .
$$

As we have shown in Section 8 any such Hamiltonian can be included into the range-2 HCB model by adding one extra second-order reduction. Thus all our results obtained for 2-local stoquastic Hamiltonians hold for $(2, k)$-local stoquastic Hamiltonians.

\section{Proof of the main theorems}

Now we have all ingredients needed for the proof of Theorems 1,2. Consider a target Hamiltonian $H \in \operatorname{StoqLH}(n, J)$ or $H \in \operatorname{StoqLH}^{*}(n, J)$. Let us prove that $H$ can be simulated with an arbitrarily small error $(\eta, \epsilon)$, by a TIM Hamiltonian $H^{\prime} \in \operatorname{TIM}\left(n^{\prime}, J^{\prime}\right)$ such that $n^{\prime} \leq \operatorname{poly}(n)$ and $J^{\prime} \leq \operatorname{poly}\left(n, J, \epsilon^{-1}, \eta^{-1}\right)$. Here we use the definition of simulation given in Section 3 . The parameters $\eta, \epsilon$ will be specified later. Indeed, consider the sequence of perturbative reductions constructed in Sections 5-11. It can be described by a sequence of Hamiltonians $H_{1}, H_{2}, \ldots, H_{R}$ and encodings $\mathcal{E}_{1}, \mathcal{E}_{2}, \ldots, \mathcal{E}_{R-1}$ such that the Hamiltonian $H_{t}$ and the encoding $\mathcal{E}_{t}$ simulate $H_{t+1}$ with a small error $\left(\eta_{t}, \epsilon_{t}\right)$ for each $t=1, \ldots, R-1$. Here $H_{R}=H$ is the desired target Hamiltonian and $H_{1}=H^{\prime}$ is a TIM Hamiltonian. Choose a simulation error $\eta_{t}=\eta / 2 R$ and and $\epsilon_{t}=\epsilon / 2 R$ for each each individual reduction. Since $R=O(1)$, this implies $\eta_{t}=\Omega(\eta)$ and $\epsilon_{t}=\Omega(\epsilon)$. By construction, each Hamiltonian $H_{t}$ belongs to one of the classes defined in Table 2 with some number of nodes (qubits) $n_{t}$ and some interaction strength $J_{t}$. In addition, each Hamiltonian $H_{t}$ (except for $H_{R}$ ) is a sum of a strong unperturbed part $\left(H_{t}\right)_{0}$ with a spectral gap $\Delta_{t} \gg\left\|H_{t+1}\right\|$ and a weak perturbation. We have shown that each reduction satisfies

$$
n_{t} \leq \operatorname{poly}\left(n_{t+1}\right) \quad \text { and } \quad J_{t} \leq \operatorname{poly}\left(n_{t+1}, J_{t+1}, \epsilon^{-1}, \eta^{-1}\right)
$$

where $t=1, \ldots, R-1$. Using the initial conditions $n_{R}=n, J_{R}=J$, and taking into account that $R=O(1)$, we conclude that $n^{\prime} \equiv n_{1} \leq \operatorname{poly}(n)$ and $J^{\prime} \equiv J_{1} \leq \operatorname{poly}\left(n, J, \epsilon^{-1}, \eta^{-1}\right)$. 
Let $\mathcal{E}=\mathcal{E}_{1} \mathcal{E}_{2} \cdots \mathcal{E}_{R-1}$ be the composition of all individual encodings. Applying Lemma 3 one infers that the Hamiltonian $H_{1}$ and the encoding $\mathcal{E}$ simulate $H_{R}$ with an error $(\tilde{\eta}, \tilde{\epsilon})$, where

$$
\tilde{\eta} \leq \frac{\eta}{2}+O(\epsilon) \max _{t} \Delta_{t}^{-1} \quad \text { and } \quad \tilde{\epsilon} \leq \frac{\epsilon}{2}+O(\epsilon) \max _{t} \frac{\left\|H_{t+1}\right\|}{\Delta_{t}}
$$

Increasing, if necessary, the spectral gaps $\Delta_{t}$ by a factor poly $\left(\eta^{-1}\right)$ one can achieve $\tilde{\eta} \leq \eta$ and $\tilde{\epsilon} \leq \epsilon$.

To prove Theorem 1 we choose $\epsilon$ as the precision specified in the statement of the theorem. The parameter $\eta$ does not play any role here. We have to use all reductions described in Sections 5-11 except for the one of Sections 8 (the latter generates multi-particle interactions that are only needed for the proof of Theorem 2). Then $H_{1}$ is a TIM Hamiltonian with interactions of degree-3. Lemma 1 implies that the $i$-th smallest eigenvalues of $H_{1}$ and $H_{R}$ differ at most by $\epsilon$ for all $i=1, \ldots, 2^{n}$. This proves Theorem 1 with $H^{\prime}=H_{1}$.

To prove Theorem 2 we shall choose $\epsilon \leq \delta / 3$, where $\delta$ is the spectral gap of $H$. We have to use all reductions described in Sections 6-11. By construction, the encodings $\mathcal{E}_{t}$ used in all these reductions map basis vectors to basis vectors. In addition, one can efficiently compute the action of $\mathcal{E}_{t}$ and $\mathcal{E}_{t}^{\dagger}$ on any basis vector. Thus the same properties hold for the full encoding $\mathcal{E}=\mathcal{E}_{1} \mathcal{E}_{2} \cdots \mathcal{E}_{R-1}$. Lemma 1 implies that the Hamiltonian $H_{1}$ has a non-degenerate ground state and a spectral gap at least $\delta-2 \epsilon \geq \delta / 3$. Let $|g\rangle$ and $\left|g^{\prime}\right\rangle$ be the ground states of $H$ and $H_{1}$. By Lemma 2,

$$
\|\left|g^{\prime}\right\rangle-\mathcal{E}|g\rangle \| \leq \eta+C \delta^{-1} \epsilon
$$

for some constant coefficient $C$. Choosing $\epsilon=\min \left(\delta / 3, \eta \delta C^{-1}\right)$ one can achieve $\|\left|g^{\prime}\right\rangle-\mathcal{E}|g\rangle \| \leq 2 \eta$. Since $\eta$ can be arbitrarily small, this proves Theorem 2 with $H^{\prime}=H_{1}$.

Let us remark that the map $H \rightarrow H^{\prime}$ in Theorem 2 can be made sufficiently smooth. More precisely, suppose $H$ smoothly depends on some parameter $\tau$ such that the $j$-th derivative of $H$ with respect to $\tau$ has norm at most poly $(n)$ for any constant $j$. Then we claim that the $j$-th derivative of $H^{\prime}$ with respect to $\tau$ has norm at most poly $\left(n, \delta^{-1}\right)$. Indeed, suppose $H_{\text {target }}=H_{t+1}$ and $H_{\text {sim }}=H_{t}$ are the target and the simulator Hamiltonians used in some individual reduction and $\left(\eta_{t}, \epsilon_{t}\right)$ is the desired simulation error. For concreteness, consider the reduction of Section 11. Note that the derivative of $H_{\text {sim }}$ becomes infinite if some of the coefficients $p_{\alpha}$ in Eq. (105) becomes zero since $H_{\text {sim }}$ contains terms proportional to $p_{\alpha}^{1 / 3}$ and $p_{\alpha}^{2 / 3}$. To avoid such singularities, let us choose a sufficiently small cutoff value $p_{\text {min }}$ and replace $p_{\alpha}$ by $\tilde{p}_{\alpha}=\sqrt{p_{\alpha}^{2}+p_{\min }^{2}}$ in Eq. (105) (recall that the coefficients $p_{\alpha}$ must be nonnegative). This gives a new target Hamiltonian $\tilde{H}_{\text {target }}$ such that $\left\|H_{\text {target }}-\tilde{H}_{\text {target }}\right\| \leq p_{\min }$ poly $(n)$. We choose $p_{\min }$ small enough so that $\left\|H_{\text {target }}-\tilde{H}_{\text {target }}\right\| \ll \epsilon_{t}$. Let $\tilde{H}_{\text {sim }}$ be the simulator Hamiltonian constructed for $\tilde{H}_{\text {target }}$. Then $\tilde{H}_{\text {sim }}$ simulates $H_{\text {target }}$ with the error approximately $\left(\eta_{t}, \epsilon_{t}\right)$ and the $j$-th derivative of $\tilde{H}_{\text {sim }}$ has norm at most $\operatorname{poly}\left(n, t_{\text {min }}^{-1}\right)=\operatorname{poly}\left(n, \epsilon_{t}^{-1}\right)=\operatorname{poly}\left(n, \delta^{-1}\right)$. By introducing a similar cutoff in all remaining reductions one can easily check that the $j$-th derivative of $H^{\prime}$ with respect to $\tau$ has norm at most poly $\left(n, \delta^{-1}\right)$. It is known that an adiabatic path with the minimum spectral gap $\delta$ such that the $j$-th derivative has norm at most $C_{j}$ can be traversed in time $T=O\left(C_{1} \delta^{-2}+C_{2} \delta^{-2}+C_{1}^{2} \delta^{-3}\right)$, see [25]. This implies Corollary 4. 
Finally, let us remark that Theorem 2 can be extended to TIM Hamiltonians with interactions of degree-3, although the corresponding encoding $\mathcal{E}$ would no longer map basis vectors to basis vectors. Indeed, let us modify the above proof of Theorem 2 by including the reduction of Section 5. Then the final TIM Hamiltonian $H^{\prime}$ has interactions of degree-3. Let $\mathcal{E}_{1}$ be the encoding used in the reduction of Section 5. Recall that $\mathcal{E}_{1}$ encodes each qubit $u$ of the target model into a onedimensional chain $L_{u}$ with a Hamiltonian $H_{\text {chain }}=-\sum_{j \in \mathbb{Z}_{m}} g Z_{j} Z_{j+1}+X_{j}$, where $m \leq \operatorname{poly}(n)$ and $g \approx 1$, see Section 5. Basis states of the logical qubit are $|\overline{0}\rangle \sim\left|\psi_{0}\right\rangle+\left|\psi_{1}\right\rangle$ and $|\overline{1}\rangle \sim\left|\psi_{0}\right\rangle-\left|\psi_{1}\right\rangle$, where $\psi_{0}$ and $\psi_{1}$ are the ground states of $H_{\text {chain }}$ satisfying $X^{\otimes m} \psi_{0,1}= \pm \psi_{0,1}$. Accordingly, the full encoding $\mathcal{E}=\mathcal{E}_{1} \mathcal{E}_{2} \cdots \mathcal{E}_{R-1}$ maps any basis vector to a tensor product of the states $|\overline{0}\rangle$ and $|\overline{1}\rangle$. Let us argue that the logical qubits can be efficiently initialized and measured. Choose any physical qubit $i \in L_{u}$. Using Eqs. $(43,44,45)$ one gets $\left\langle\overline{0}\left|Z_{i}\right| \overline{0}\right\rangle=\xi$ and $\left\langle\overline{1}\left|Z_{i}\right| \overline{1}\right\rangle=-\xi$, where $\xi \geq \operatorname{poly}\left(n^{-1}\right)$. Thus one can measure the logical qubit $u$ in the $Z$-basis by measuring any physical qubit of $L_{u}$ in the $Z$-basis. However, the measurement has to be repeated poly $(n)$ times to get a reliable statistics. One can measure the logical qubit in the $X$-basis in a single shot by measuring every qubit of $L_{u}$ in the $X$-basis. Computing the product of the measured outcomes gives the eigenvalue of $X^{\otimes m}$ which differentiates between $\psi_{0}$ and $\psi_{1}$. Finally, the state $|\overline{\mid}\rangle=\left|\psi_{0}\right\rangle$ can be prepared by the adiabatic evolution starting from the product state $\left|+{ }^{\otimes m}\right\rangle$ and adiabatically turning on the parameter $g$ in the Hamiltonian $H_{\text {chain }}$. It is well-known that the minimum spectral gap of $H_{\text {chain }}$ is $\Omega\left(\mathrm{m}^{-1}\right)$, so that the initialization can be done in time poly $(n)$. The logical state $|\overline{0}\rangle$ can be obtained from $|\bar{\mp}\rangle$ by adiabatically changing the logical Hamiltonian from $-\bar{X}$ to $-\bar{Z}$.

\section{A Bounds on the energy splitting and matrix elements for the Ising chain}

In this section we prove Eqs. $(41,45)$.

Let us first prove Eq. (41). Choose any $g^{-1}<R<1$ and consider contours

$$
C=\left\{z \in \mathbb{C}^{2}:|z|=R\right\} \quad \text { and } \quad C^{-1}=\left\{z \in \mathbb{C}^{2}:|z|=R^{-1}\right\}
$$

We orient $C$ and $C^{-1}$ clockwise and counter-clockwise respectively. Denote $E_{+} \equiv E_{0}$ and $E_{-} \equiv E_{1}$, see Fact 1. Using Eq. (38) one can easily check that

$$
E_{ \pm}=\frac{m}{2 \pi i} \oint_{C \cup C^{-1}} \frac{d z \sqrt{g^{2}+1-g\left(z+z^{-1}\right)}}{z\left(z^{m} \mp 1\right)} .
$$

Since the contours $C$ and $C^{-1}$ can be mapped to each other via a change of variable $z \rightarrow z^{-1}$, one gets

$$
E_{ \pm}=-\frac{m}{2 \pi i} \oint_{C} \frac{d z\left(1 \pm z^{m}\right) \sqrt{g^{2}+1-g\left(z+z^{-1}\right)}}{z\left(z^{m} \mp 1\right)} .
$$

Therefore

$$
\delta=E_{-}-E_{+}=-\frac{2 m}{\pi i} \oint_{C} \frac{d z z^{m-1} \sqrt{g^{2}+1-g\left(z+z^{-1}\right)}}{1-z^{2 m}} .
$$


The function $\sqrt{g^{2}+1-g\left(z+z^{-1}\right)}$ is analytic in the complex plane with cuts along the intervals $\left[0, g^{-1}\right]$ and $[g, \infty]$. Deforming the contour $C$ such that it goes from 0 to $g^{-1}$ in the upper half-plane and then returns to 0 in the lower half-plane one gets

$$
\delta=\frac{4 m \sqrt{g}}{\pi} \int_{0}^{g^{-1}} \frac{x^{m-1} \sqrt{x+x^{-1}-g-g^{-1}} d x}{1-x^{2 m}} .
$$

Using a bound

$$
x+x^{-1}-g-g^{-1}=x^{-1}(g-x)\left(g^{-1}-x\right) \geq\left(g-g^{-1}\right) x^{-1}\left(g^{-1}-x\right)
$$

we obtain

$$
\delta \geq \frac{4 m \sqrt{g^{2}-1}}{\pi} \int_{0}^{g^{-1}} x^{m-3 / 2} \sqrt{g^{-1}-x} d x .
$$

Making a change of variables $x=g^{-1} y$ one gets

$$
\delta \geq \frac{4 m g^{-m} \sqrt{g^{2}-1}}{\pi} \int_{0}^{1} d y y^{m-3 / 2} \sqrt{1-y} \geq \Omega(1) g^{-m} m^{-1} \sqrt{g^{2}-1} .
$$

Here we noted that the integral over $y$ is equal to the beta function $B(m-1 / 2,3 / 2)=\Omega\left(m^{-2}\right)$. Finally, since $\sqrt{g^{2}-1}=\Omega\left(m^{-1 / 2}\right)$, one gets $\delta \geq \Omega\left(m^{-c-3 / 2}\right)$. To get an upper bound in Eq. (41) we note that

$$
x^{-1}(g-x)\left(g^{-1}-x\right) \leq g x^{-1}\left(g^{-1}-x\right) \quad \text { and } \quad\left(1-x^{2 m}\right)^{-1} \leq\left(1-g^{-2 m}\right)^{-1} \leq O(1) .
$$

Performing the same change of variable as above one gets

$$
\delta \leq O\left(m g^{-m+1}\right) \int_{0}^{1} d y y^{m-3 / 2} \sqrt{1-y}=O\left(m g^{-m+1}\right) B(m-1 / 2,3 / 2)=O\left(m^{-c-1 / 2}\right) .
$$

Let us now prove Eq. (45). We shall use the notations of Fact 2. In the limit $g \rightarrow \infty$ the Hamiltonian Eq. (36) has ground states $\left|0^{\otimes m}\right\rangle$ and $\left|1^{\otimes m}\right\rangle$, that is,

$$
\left|\psi_{0}\right\rangle=\frac{1}{\sqrt{2}}\left(\left|0^{\otimes m}\right\rangle+\left|1^{\otimes m}\right\rangle\right) \quad \text { and } \quad\left|\psi_{1}\right\rangle=\frac{1}{\sqrt{2}}\left(\left|0^{\otimes m}\right\rangle-\left|1^{\otimes m}\right\rangle\right) .
$$

Thus $\xi=\left\langle\psi_{1}\left|Z_{j}\right| \psi_{0}\right\rangle=1$. Since $\xi$ is a real continuous function of $g$, it suffices to show that $|\xi| \geq\left(1-g^{-2}\right)^{1 / 8}$ for all $g>1$. Let us write $|\xi|=\left(1-g^{-2}\right)^{1 / 8} \eta$. Then we have to prove that $\eta \geq 1$. Since we already know that $\eta=1$ in the limit $g \rightarrow \infty$, it suffices to show that $\eta$ is a monotone decreasing function of $g$ for all $g>1$. Below we prove that

$$
\eta^{-1} \frac{\partial \eta}{\partial g} \leq 0 \quad \text { for all } g>1
$$


Computing the derivative over $g$ one gets

$$
\dot{\epsilon_{p}}=\frac{1}{2}\left(\frac{g}{\epsilon_{p}}+\frac{\epsilon_{p}}{g}-\frac{1}{g \epsilon_{p}}\right)
$$

and thus

$$
\frac{\partial}{\partial g} \sum_{p} \sum_{q} \log \left(\epsilon_{p}+\epsilon_{q}\right)=\frac{m^{2}}{2 g}+\frac{1}{2}\left(g-g^{-1}\right)\left(\sum_{p} \epsilon_{p}^{-1}\right)\left(\sum_{q} \epsilon_{q}^{-1}\right) .
$$

Here the sums over $p$ and $q$ can range over either $\mathbb{Z}_{m}$ or $\mathbb{Z}_{m}+1 / 2$. Using Eq. (127) one gets

$$
\eta^{-1} \frac{\partial \eta}{\partial g}=\frac{1}{16}\left(g^{-1}-g\right)\left(Z_{+}-Z_{-}\right)^{2},
$$

where

$$
Z_{+} \equiv \sum_{p \in \mathbb{Z}_{m}} \epsilon_{p}^{-1} \quad \text { and } \quad Z_{-} \equiv \sum_{q \in \mathbb{Z}_{m}+1 / 2} \epsilon_{q}^{-1}
$$

This implies Eq. (126) and proves that $\eta \geq 1$ for all $g>1$.

Acknowledgments - The authors would like to thank Barbara Terhal for helpful discussions on perturbative reductions. S.B. acknowledges NSF Grant CCF-1110941.

\section{References}

[1] A. Kitaev, A. Shen, and M. Vyalyi. Classsical and quantum computation. American Mathematical Soc., 2002.

[2] R. Oliveira and B. M. Terhal. The complexity of quantum spin systems on a two-dimensional square lattice. Quant. Inf. and Comp., 8(10):900-924, 2008.

[3] D. Aharonov, D. Gottesman, S. Irani, and J. Kempe. The power of quantum systems on a line. Comm. Math. Phys., 287(1):41-65, 2009.

[4] S. Hallgren, D. Nagaj, and S. Narayanaswami. The Local Hamiltonian problem on a line with eight states is QMA-complete. Quant. Inf. and Comp., 13(9-10):721-750, 2013.

[5] N. Schuch and F. Verstraete. Computational complexity of interacting electrons and fundamental limitations of density functional theory. Nature Physics, 5(10):732-735, 2009.

[6] A. M. Childs, D. Gosset, and Z. Webb. The Bose-Hubbard model is QMA-complete. preprint arXiv:1311.3297, 2013. 
[7] T. Cubitt and A. Montanaro. Complexity classification of local Hamiltonian problems. preprint arXiv:1311.3161, 2013.

[8] S. Bravyi, D. DiVincenzo, R. Oliveira, and B. Terhal. The complexity of stoquastic local Hamiltonian problems. Quant. Inf. Comp., 8(5):0361-0385, 2008.

[9] M. Suzuki, S. Miyashita, and A. Kuroda. Monte Carlo simulation of quantum spin systems. I. Prog. Theor. Phys., 58(5):1377-1387, 1977.

[10] N. Prokof'ev, B. Svistunov, and I. Tupitsyn. Exact quantum Monte Carlo process for the statistics of discrete systems. Pis'ma v Zh.Eks.Teor.Fiz., 64(12):911-916, 1996.

[11] A. W. Sandvik and J. Kurkijärvi. Quantum Monte Carlo simulation method for spin systems. Phys. Rev. B, 43(7):5950, 1991.

[12] N. Trivedi and D.M. Ceperley. Green-function Monte Carlo study of quantum antiferromagnets. Phys. Rev. B, 40(4):2737, 1989.

[13] S. Bravyi. Monte Carlo simulation of stoquastic Hamiltonians. arXiv preprint 1402.2295, 2014.

[14] P. Pfeuty. The one-dimensional Ising model with a transverse field. Ann. of Phys., 57(1):79-90, 1970.

[15] Subir Sachdev. Quantum Phase Transitions. Cambridge University Press, 2007.

[16] T.K. Kopeć, K.D. Usadel, and G. Büttner. Instabilities in the quantum Sherrington-Kirkpatrick Ising spin glass in transverse and longitudinal fields. Phys. Rev. B, 39(16):12418, 1989.

[17] C. Laumann, A. Scardicchio, and S.L. Sondhi. Cavity method for quantum spin glasses on the Bethe lattice. Phys. Rev. B, 78(13):134424, 2008.

[18] E. Farhi, J. Goldstone, S. Gutmann, and M. Sipser. Quantum computation by adiabatic evolution. arXiv preprint quant-ph/0001106, 2000.

[19] S. Boixo, T. Rønnow, S. Isakov, Z. Wang, D. Wecker, D. Lidar, J. Martinis, and M. Troyer. Evidence for quantum annealing with more than one hundred qubits. Nature Physics, 10(3):218$224,2014$.

[20] T. F. Rønnow, Z. Wang, J. Job, S. Boixo, S. Isakov, D. Wecker, J. Martinis, D. Lidar, and M. Troyer. Defining and detecting quantum speedup. arXiv preprint 1401.2910, 2014.

[21] S. W. Shin, G. Smith, J. Smolin, and U. Vazirani. How "quantum" is the D-wave machine? arXiv preprint 1401.7087, 2014.

[22] J. Kempe, A. Kitaev, and O. Regev. The complexity of the local Hamiltonian problem. SIAM J. on Comp., 35(5):1070-1097, 2006. 
[23] S. Bravyi, A. J. Bessen, and B. M. Terhal. Merlin-Arthur games and stoquastic complexity. arXiv preprint quant-ph/0611021, 2006.

[24] E. Farhi, J. Goldstone, S. Gutmann, J. Lapan, A. Lundgren, and D. Preda. A quantum adiabatic evolution algorithm applied to random instances of an NP-complete problem. Science, 292(5516):472-475, 2001.

[25] S. Jansen, M.-B. Ruskai, and R. Seiler. Bounds for the adiabatic approximation with applications to quantum computation. J. Math. Phys., 48:102111, 2007.

[26] J.R. Schrieffer and P.A. Wolff. Relation between the Anderson and Kondo hamiltonians. Phys. Rev., 149(2):491, 1966.

[27] S. Bravyi, D. P. DiVincenzo, D. Loss, and B. M. Terhal. Quantum simulation of many-body Hamiltonians using perturbation theory with bounded-strength interactions. Phys. Rev. Lett., 101(7):070503, 2008.

[28] S. Bravyi, D. P. DiVincenzo, and D. Loss. Schrieffer-Wolff transformation for quantum manybody systems. Ann. of Phys., 326(10):2793-2826, 2011.

[29] G. von Gehlen, N. Iorgov, S. Pakuliak, V. Shadura, and Yu. Tykhyy. Form-factors in the BaxterBazhanov-Stroganov model II: Ising model on the finite lattice. J. of Phys. A: Mathematical and Theoretical, 41(9):095003, 2008.

[30] S. Isakov, M. B. Hastings, and R. Melko. Topological entanglement entropy of a Bose-Hubbard spin liquid. Nature Physics, 7(10):772-775, 2011.

[31] S. Isakov, R. Melko, and M. B. Hastings. Universal signatures of fractionalized quantum critical points. Science, 335(6065):193-195, 2012.

[32] Y. Cao and D. Nagaj. Perturbative gadgets without strong interactions. preprint arXiv:1408.5881, 2014.

[33] M. B. Hastings. Obstructions to classically simulating the quantum adiabatic algorithm. Quant. Inf. and Comp., 13(11-12):1038-1076, 2013.

[34] P. Fendley. Free parafermions. arXiv preprint arXiv:1310.6049, 2013. 